Katarzyna Steliga and Dominik Szynal (Lublin)

\title{
ON COUNTING DISTRIBUTIONS RELATED TO THE DELAPORTE DISTRIBUTION
}

Abstract. We introduce the $\alpha$-modified negative binomial distribution and the $\alpha$-modified Delaporte distribution. The Delaporte distribution is a member of the class of $\alpha$-modified Delaporte distributions. The probability distributions studied can be applied e.g. in actuarial sciences. The main result of the paper shows that the $\alpha$-modified negative binomial distribution fits the number of automobile insurance claims better than the negative binomial distribution, while the $\alpha$-modified Delaporte distribution describes the number of car accidents better than the classical Delaporte distribution. Characteristics of the distributions considered (moments, coefficient of variation, skewness and kurtosis) are computed. Moreover, we study some compounds of the $\alpha$-modified negative binomial and $\alpha$-modified Delaporte distributions. We also study the compound $\alpha$-modified Delaporte distribution with Borel summands and gamma summands. Characteristics (moments, coefficient of variation, skewness and kurtosis) of these distributions are computed. Moreover, the compound $\alpha$-modified negative binomial distribution with gamma summands is investigated.

1. Introduction. Berg and Jaworski [BJ] introduced the $\alpha$-modified binomial and $\alpha$-modified Poisson distributions with applications in random mapping theory. Some generalisations of those distributions were studied in Chakraborty [C]. We introduce the $\alpha$-modified geometric, $\alpha$-modified negative binomial and $\alpha$-modified Delaporte distribution proposing some of their applications. In Section 4 we give formulae for moments of the Borel distribution and we complete the investigations of the compound Delaporte

2010 Mathematics Subject Classification: Primary 60E10; Secondary 62E99.

Key words and phrases: $\alpha$-modified Poisson distribution, $\alpha$-modified negative binomial distribution, $\alpha$-modified Delaporte distribution, characteristics, compound distributions. Received 9 February 2017; revised 25 June 2018.

Published online 1 February 2019. 
distribution with Borel summands discussed by Finner et al. [FKS. Section 5 is devoted to the compound $\alpha$-modified Delaporte distribution with Borel and gamma summands. Section 6 concerns the compound $\alpha$-modified negative binomial distribution where the i.i.d. summands have a gamma distribution.

First we recall the definitions of the counting distributions used in this paper. The geometric distribution $\mathrm{G}(q)$ is given by

$$
P[X=x]=q^{x}(1-q), \quad x \in \mathbb{N}_{0}=\mathbb{N} \cup\{0\} ; 0<q<1,
$$

the negative binomial distribution $\mathrm{NB}(t, q)$ by

$$
P[X=x]=\left(\begin{array}{c}
x+t-1 \\
x
\end{array}\right) q^{x}(1-q)^{t}, \quad x \in \mathbb{N}_{0} ; 0<q<1, t>0,
$$

the Poisson distribution $\mathrm{P}(\lambda)$ by

$$
P[X=x]=\frac{\lambda^{x}}{x !} e^{-\lambda}, \quad x \in \mathbb{N}_{0} ; \lambda>0,
$$

and the Delaporte distribution $\operatorname{Del}(t, \beta, \lambda)$ by

$P[X=x]=\sum_{i=0}^{x} \frac{\Gamma(t+i) \beta^{i} \lambda^{x-i}}{\Gamma(t) i !(1+\beta)^{t+i}(x-i) !} e^{-\lambda}, \quad x \in \mathbb{N}_{0} ; \lambda>0, \beta>0, t>0$ (see [V], p. 619]).

2. $\alpha$-Modified negative binomial distribution. It is known that the classical negative binomial distribution is generated in the following way.

Suppose that given $\Lambda=\lambda$ a random variate $Y$ is Poisson distributed (1.3), and $\Lambda$ is a two-parameter gamma distribution with the probability density function (pdf)

$$
f_{\Lambda}(\lambda)=\left[\beta^{t} \Gamma(t)\right]^{-1} \lambda^{t-1} \exp (-\lambda / \beta), \quad \lambda>0 ; \beta>0, t>0 .
$$

Then the mixed Poisson distribution with the gamma mixing is the negative binomial distribution 1.2$)(X \sim \mathrm{NB}(t, q)$, where $q=\beta /(1+\beta))$ (see [DM, KPW, p. 212]).

Using the above idea we introduce the $\alpha$-modified negative binomial distribution. To this end we use the $\alpha$-modified Poisson distribution ( $X \sim$ $\operatorname{MP}(\psi, \lambda))$ introduced by Berg and Jaworski [BJ]:

$$
P[X=x]=\frac{(\lambda+\alpha \psi)^{x}}{x !}(1-\psi) e^{-\lambda}, \quad x=0,1, \ldots ;
$$

$\lambda>0$ and $\psi$ are parameters such that $|\psi|<1$ and $\lambda+\psi \geq 0 ; \alpha$ is Riordan's symbol, where $\alpha^{k} \equiv \alpha_{k}=k$ ! (see [R, p. 21]). 
Let the extended umbral numbers $(\alpha(t))^{l}$ be defined as follows:

$$
(\alpha(t))^{l}= \begin{cases}\left(\begin{array}{c}
l+t-1 \\
l
\end{array}\right) l ! & \text { for } t>0, l \in \mathbb{N}_{0}, \\
0 & \text { for } t=0\end{cases}
$$

or

$$
(\alpha(t))^{l}=\frac{\Gamma(t+l)}{\Gamma(t)}=t^{[l]}, \quad t>0, l \in \mathbb{N}_{0},
$$

where $t^{[l]}$ is the $l$ th rising factorial power: $t^{[l]}=t(t+1) \cdot \ldots \cdot(t+l-1)$ (see [W, p. 25]).

For $t=j, j \in \mathbb{N}_{0}$ in $(2.3)$ see [BN, C, FKS, J] and [R, p. 23].

We have the following result.

Theorem 2.1. Let $Y$ have the $\alpha$-modified Poisson distribution $(Y \sim$ $\operatorname{MP}(\psi, \lambda))$ as in (2.2). Suppose that $\Lambda=\lambda$ is two-parameter gamma distributed with the probability density function (2.1). Then

$$
\begin{array}{r}
P[X=x]=E[P[Y=x \mid \Lambda=\lambda]]=\frac{(\psi \alpha(1)+q \alpha(t))^{x}}{x !}(1-\psi)(1-q)^{t}, \\
x=0,1, \ldots ;
\end{array}
$$

$t, \psi$ and $q$ are parameters such that $t>0,|\psi|<1,0<q<1, \psi+q>0$, and $(\alpha(t))^{l}$ is as in $(2.3)$.

Proof. We consider the mixed $\alpha$-modified Poisson distribution with $\Lambda$ the gamma mixing distribution (2.1). To give the probability function of that mixture we start with

$$
P[X=x]=(1-\psi)\left[\beta^{t} \Gamma(t) x !\right]^{-1} \int_{0}^{\infty} \lambda^{t-1} \exp [-\lambda(\beta+1) / \beta](\lambda+\alpha \psi)^{x} d \lambda .
$$

Now we have

$$
\sum_{k=0}^{x}\left(\begin{array}{l}
x \\
k
\end{array}\right) \lambda^{k}(x-k) ! \psi^{x-k}=(\lambda+\alpha \psi)^{x}
$$

Hence

$$
\begin{aligned}
& P[X=x] \\
& =(1-\psi)\left[\beta^{t} \Gamma(t) x !\right]^{-1} \sum_{k=0}^{x}\left(\begin{array}{l}
x \\
k
\end{array}\right)(x-k) ! \psi^{x-k} \int_{0}^{\infty} \lambda^{t+k-1} \exp [-\lambda(1+\beta) / \beta] d \lambda \\
& =(1-\psi)\left[\beta^{t} \Gamma(t) x !\right]^{-1} \sum_{k=0}^{x}\left(\begin{array}{l}
x \\
k
\end{array}\right)(x-k) ! \psi^{x-k}\left(\frac{\beta}{1+\beta}\right)^{t+k} \Gamma(t+k) .
\end{aligned}
$$


In terms of the extended umbral numbers $(\alpha(t))^{l}$ of 2.4 we get

$$
\begin{aligned}
P[X=x] & =\frac{1-\psi}{x !}(1+\beta)^{-t} \sum_{k=0}^{x}\left(\begin{array}{l}
x \\
k
\end{array}\right)(x-k) ! \psi^{x-k}(\alpha(t))^{k}(\beta /(1+\beta))^{k} \\
& =\frac{\left(\psi \alpha(1)+\beta(1+\beta)^{-1} \alpha(t)\right)^{x}}{x !}(1-\psi)(1+\beta)^{-t} \\
& =\frac{(\psi \alpha(1)+q \alpha(t))^{x}}{x !}(1-\psi)(1-q)^{t} .
\end{aligned}
$$

Remark 2.2. Formula (2.5) with $\psi=0$ takes the form

$$
P[X=x]=\frac{(q \alpha(t))^{x}}{x !}(1-q)^{t} .
$$

It is another form of the classical negative binomial distribution 1.2.

Definition 2.3. We say that $X$ has the $\alpha$-modified negative binomial distribution $(X \sim \operatorname{MNB}(\psi, t, q))$ if its probability function is given by (2.5).

The probability function (2.5) with $t=1$,

$$
P[X=x]=\frac{(\alpha \psi+\alpha q)^{x}}{x !}(1-\psi)(1-q), \quad x=0,1, \ldots,
$$

is called the $\alpha$-modified geometric distribution $(X \sim \operatorname{MG}(\psi, q))$.

Formula 2.6 can be written as follows:

$$
\begin{aligned}
& P[X=x]=(1-\psi)(1-q) \frac{q^{x+1}-\psi^{x+1}}{q-\psi} \\
& =(1-\psi)(1-q)\left(q^{x}+\sum_{\nu=1}^{x} \psi^{\nu} q^{x-\nu}\right), \quad x=0,1, \ldots ; \psi+q>0,
\end{aligned}
$$

which can be treated as two-parameter geometric distribution (we call it the generalised geometric distribution).

REMARK 2.4. Suppose that $X$ has the geometric distribution (1.1), $q:=$ $\psi, 0<\psi<1$ and $Y$ has the Poisson distribution (1.3). If $X$ and $Y$ are independent, then

$$
P[Z=X+Y=z]=\frac{(\lambda+\alpha \psi)^{z}}{z !}(1-\psi) e^{-\lambda}
$$

(see $[\mathrm{BJ}]$ ).

A generalisation of this result is as follows.

Suppose that $X$ has the probability function (2.7) and $Y$, independent of $X$, has the Poisson distribution (1.3). Then

$$
P[Z=X+Y=z]=(1-\psi)(1-q) \frac{q(\lambda+\alpha q)^{z}-\psi(\lambda+\alpha \psi)^{z}}{z !(q-\psi)} e^{-\lambda}
$$

and $\lim _{q \rightarrow 0} P[Z=z]$ is given by $(2.8)$. 
We denote by

- ${ }_{\alpha} m_{(r)}:={ }_{\alpha} E X(X-1) \cdot \ldots \cdot(X-r+1)$ the $r$ th factorial moment,

- ${ }_{\alpha} m_{r}:={ }_{\alpha} E X^{r}$ the $r$ th uncorrected moment, for short, the $r$ th moment,

- ${ }_{\alpha} \mu_{r}:={ }_{\alpha} E(X-E X)^{r}$ the $r$ th central moment,

- $t^{[n]}=t \cdot(t+1) \cdot \ldots \cdot(t+n-1)$ the $n$th rising factorial of $t$,

- $r_{(k)}=r \cdot(r-1) \cdot \ldots \cdot(r-k+1)$ the $k$ th falling factorial of $r$ (see [W, p. 3]).

THEOREM 2.5. The probability generating function, the rth factorial moment, the rth moment and the rth central moment of the $\alpha$-modified negative binomial distribution (2.5) are as follows:

$$
\begin{aligned}
{ }_{\alpha} G_{X}(s) & =(1-\psi)(1-q)^{t} e^{s(\psi \alpha(1)+q \alpha(t))} \\
{ }_{\alpha} m_{(r)} & =\left(\alpha(t) \frac{q}{1-q}+\alpha(1) \frac{\psi}{1-\psi}\right)^{r} \\
& =\sum_{m=0}^{r} r_{(m)} t^{[r-m]}\left(\frac{q}{1-q}\right)^{r-m}\left(\frac{\psi}{1-\psi}\right)^{m} \\
{ }_{\alpha} m_{r} & =\sum_{k=0}^{r} S(r, k)\left(\alpha(t) \frac{q}{1-q}+\alpha(1) \frac{\psi}{1-\psi}\right)^{k} \\
& =\sum_{k=0}^{r} S(r, k) \sum_{m=0}^{k} k_{(m)} t^{[k-m]}\left(\frac{q}{1-q}\right)^{k-m}\left(\frac{\psi}{1-\psi}\right)^{m}
\end{aligned}
$$

and

$$
\begin{aligned}
{ }_{\alpha} \mu_{r} & =\sum_{l=0}^{r}\left(\begin{array}{l}
r \\
l
\end{array}\right)\left(-{ }_{\alpha} m_{1}\right)^{r-l} \sum_{k=0}^{l} S(l, k)\left(\alpha(t) \frac{q}{1-q}+\alpha(1) \frac{\psi}{1-\psi}\right)^{k} \\
& =\sum_{l=0}^{r}\left(\begin{array}{l}
r \\
l
\end{array}\right)\left(-{ }_{\alpha} m_{1}\right)^{r-l} \sum_{k=0}^{l} S(l, k) \sum_{m=0}^{k} k_{(m)} t^{[k-m]}\left(\frac{q}{1-q}\right)^{k-m}\left(\frac{\psi}{1-\psi}\right)^{m},
\end{aligned}
$$

where ${ }_{\alpha} m_{1}=\left(\alpha(t) \frac{q}{1-q}+\alpha(1) \frac{\psi}{1-\psi}\right)^{1}=t \frac{q}{1-q}+\frac{\psi}{1-\psi}$ and $S(n, k)$ is the Stirling number of the second kind.

Proof. Using the pgf of a non-negative integer-valued random variable $X$,

$$
G_{X}(s)=E s^{X}=\sum_{n=0}^{\infty} s^{n} \cdot P[X=n], \quad|s|<1
$$

(see [G, p. 186]), and taking into account 2.5 we obtain 2.9 which we can write as follows:

$$
{ }_{\alpha} G_{X}(s)=(1-\psi)(1-q)^{t} e^{s(\psi \alpha(1)+q \alpha(t))}=\frac{1-\psi}{1-s \psi}\left(\frac{1-q}{1-s q}\right)^{t} .
$$


The $k$ th derivative of ${ }_{\alpha} G_{X}(s)$ is given by

$$
{ }_{\alpha} G_{X}^{(k)}(s)={ }_{\alpha} G_{X}(s) \sum_{i=0}^{k} k !((k-i) !)^{-1} t^{[k-i]}(q /(1-s q))^{k-i}(\psi /(1-s \psi))^{i} .
$$

Letting $s=1$ in $(2.14)$ we get the $k$ th factorial moment of the $\alpha$-modified negative binomial distribution.

Now applying the relations between the factorial moments and central moments:

$$
\begin{aligned}
E X^{r} & =\sum_{k=0}^{r} S(r, k) E X^{(k)}, \\
E(X-E X)^{r} & =\sum_{i=0}^{r}\left(\begin{array}{c}
r \\
i
\end{array}\right)(-E X)^{r-i} E X^{i} \\
& =(-E X)^{r}+\sum_{i=1}^{r}\left(\begin{array}{c}
r \\
i
\end{array}\right)(-E X)^{r-i} E X^{i}
\end{aligned}
$$

(cf. [JKK, pp. 52-53]), we are led to 2.11) and 2.12), respectively.

Corollary 2.6. If $X \sim \operatorname{MNB}(\psi, t, q)$, then

$$
\begin{aligned}
{ }_{\alpha} m_{1}= & t \frac{q}{1-q}+\frac{\psi}{1-\psi}, \\
{ }_{\alpha} m_{2}= & t \frac{q}{1-q}+t^{[2]}\left(\frac{q}{1-q}\right)^{2}+\frac{\psi}{1-\psi}\left(1+2 t \frac{q}{1-q}+\frac{2 \psi}{1-\psi}\right), \\
{ }_{\alpha} m_{3}= & t \frac{q}{1-q}+3 t^{[2]}\left(\frac{q}{1-q}\right)^{2}+t^{[3]}\left(\frac{q}{1-q}\right)^{3} \\
& +\frac{\psi}{1-\psi}\left(1+6 \frac{t}{1-\psi} \frac{q}{1-q}+\frac{6 \psi}{(1-\psi)^{2}}+3 t^{[2]}\left(\frac{q}{1-q}\right)^{2}\right), \\
{ }_{\alpha} m_{4}= & t \frac{q}{1-q}+7 t^{[2]}\left(\frac{q}{1-q}\right)^{2}+6 t^{[3]}\left(\frac{q}{1-q}\right)^{3}+t^{[4]}\left(\frac{q}{1-q}\right)^{4} \\
& +\frac{\psi}{1-\psi}\left(1+14 t \frac{q}{1-q}+36\left(\frac{\psi}{1-\psi}\right)^{2}+36 t \frac{q}{1-q} \frac{\psi}{1-\psi}\right. \\
& +18 t^{[2]}\left(\frac{q}{1-q}\right)^{2}+24\left(\frac{\psi}{1-\psi}\right)^{3}+24 t \frac{q}{1-q}\left(\frac{\psi}{1-\psi}\right)^{2} \\
& \left.+12 t^{[2]}\left(\frac{q}{1-q}\right)^{2} \frac{\psi}{1-\psi}+4 t^{[3]}\left(\frac{q}{1-q}\right)^{3}+14 \frac{\psi}{1-\psi}\right), \\
{ }_{\alpha} \mu_{2}= & t \frac{q}{(1-q)^{2}}+\frac{\psi}{(1-\psi)^{2}},
\end{aligned}
$$




$$
\begin{aligned}
{ }_{\alpha} \mu_{3}= & t \frac{q}{(1-q)^{3}}(1+q)+\frac{\psi}{(1-\psi)^{3}}(1+\psi), \\
\alpha \mu_{4}= & 3 t^{2} \frac{q^{2}}{(1-q)^{4}}+t \frac{q}{(1-q)^{4}}\left(1+q^{2}+4 q\right) \\
& +\frac{\psi}{(1-\psi)^{4}}\left[1+7 \psi+\psi^{2}+6 t \frac{q}{(1-q)^{2}}(1-\psi)^{2}\right]
\end{aligned}
$$

and the coefficient of variation, skewness and kurtosis are as follows:

$$
\begin{aligned}
V= & \left({ }_{\alpha} \mu_{2}\right)^{1 / 2} /{ }_{\alpha} m_{1}=\frac{\left(t q(1-\psi)^{2}+\psi(1-q)^{2}\right)^{1 / 2}}{t q(1-\psi)+\psi(1-q)}, \\
\gamma={ }_{\alpha} \mu_{3} /\left({ }_{\alpha} \mu_{2}\right)^{3 / 2}= & \frac{t q(1+q)(1-\psi)^{3}+\psi(1-q)^{3}(1+\psi)}{\left(t q(1-\psi)^{2}+\psi(1-q)^{2}\right)^{3 / 2}}, \\
\kappa= & { }_{\alpha} \mu_{4} /\left({ }_{\alpha} \mu_{2}\right)^{2}=\frac{t q\left(1+q(3 t+4)+q^{2}\right)(1-\psi)^{4}+\psi(1-q)^{4}\left(1+7 \psi+\psi^{2}\right)}{t^{2} q^{2}(1-\psi)^{4}+2 t q \psi(1-q)^{2}(1-\psi)^{2}+\psi^{2}(1-q)^{4}} \\
& +\frac{6 t q \psi(1-q)^{2}(1-\psi)^{2}}{t^{2} q^{2}(1-\psi)^{4}+2 t q \psi(1-q)^{2}(1-\psi)^{2}+\psi^{2}(1-q)^{4}} .
\end{aligned}
$$

The assumption that $\psi=0$ yields formulae for the characteristics of the classical negative binomial distribution (see [JKK, p. 216], [PW, p. 91]).

Theorem 2.5 implies the following result.

THEOREM 2.7. The probability generating function, the rth factorial moment, the rth moment and the rth central moment of the $\alpha$-modified geometric distribution 2.6 are as follows:

$$
\begin{aligned}
{ }_{\alpha} G_{X}(s) & =(1-\psi)(1-q) e^{s(\alpha \psi+\alpha q)}=\frac{1-\psi}{1-s \psi} \frac{1-q}{1-s q} \\
{ }_{\alpha} m_{(r)} & =\left(\alpha \frac{q}{1-q}+\alpha \frac{\psi}{1-\psi}\right)^{r} \\
& =r !\left[\left(\frac{q}{1-q}\right)^{r+1}-\left(\frac{\psi}{1-\psi}\right)^{r+1}\right]\left(\frac{q}{1-q}-\frac{\psi}{1-\psi}\right)^{-1}, \\
{ }_{\alpha} m_{r}= & \sum_{k=0}^{r} S(r, k)\left(\alpha \frac{q}{1-q}+\alpha \frac{\psi}{1-\psi}\right)^{k} \\
= & \sum_{k=0}^{r} S(r, k) k !\left[\left(\frac{q}{1-q}\right)^{k+1}-\left(\frac{\psi}{1-\psi}\right)^{k+1}\right]\left(\frac{q}{1-q}-\frac{\psi}{1-\psi}\right)^{-1}, \\
{ }_{\alpha} \mu_{r}= & \sum_{l=0}^{r}\left(\begin{array}{l}
r \\
l
\end{array}\right)\left(-{ }_{\alpha} m_{1}\right)^{r-l} \sum_{k=0}^{l} S(l, k)\left(\alpha \frac{q}{1-q}+\alpha \frac{\psi}{1-\psi}\right)^{k}
\end{aligned}
$$




$$
\begin{aligned}
= & \sum_{l=0}^{r}\left(\begin{array}{l}
r \\
l
\end{array}\right)\left(-{ }_{\alpha} m_{1}\right)^{r-l} \sum_{k=0}^{l} S(l, k) k !\left[\left(\frac{q}{1-q}\right)^{k+1}-\left(\frac{\psi}{1-\psi}\right)^{k+1}\right] \\
& \cdot\left(\frac{q}{1-q}-\frac{\psi}{1-\psi}\right)^{-1},
\end{aligned}
$$

where

$$
{ }_{\alpha} m_{1}=\left(\alpha \frac{q}{1-q}+\alpha \frac{\psi}{1-\psi}\right)^{1}=\frac{q}{1-q}+\frac{\psi}{1-\psi} .
$$

Corollary 2.8. If $X \sim \operatorname{MG}(\psi, q)$, then

$$
\begin{aligned}
& { }_{\alpha} m_{1}=\frac{q}{1-q}+\frac{\psi}{1-\psi}, \\
& { }_{\alpha} m_{2}=\frac{q}{1-q}+2\left(\frac{q}{1-q}\right)^{2}+\frac{\psi}{1-\psi}\left(1+2 \frac{q}{1-q}+\frac{2 \psi}{1-\psi}\right), \\
& { }_{\alpha} m_{3}=\frac{q}{1-q}+6\left(\frac{q}{1-q}\right)^{2}+6\left(\frac{q}{1-q}\right)^{3} \\
& +\frac{\psi}{1-\psi}\left(1+\frac{6}{1-\psi} \frac{q}{1-q}+\frac{6 \psi}{(1-\psi)^{2}}+6\left(\frac{q}{1-q}\right)^{2}\right), \\
& { }_{\alpha} m_{4}=\frac{q}{1-q}+14\left(\frac{q}{1-q}\right)^{2}+36\left(\frac{q}{1-q}\right)^{3}+24\left(\frac{q}{1-q}\right)^{4} \\
& +\frac{\psi}{1-\psi}\left(1+14 \frac{q}{1-q}+36\left(\frac{\psi}{1-\psi}\right)^{2}+36 \frac{q}{1-q} \frac{\psi}{1-\psi}\right. \\
& +36\left(\frac{q}{1-q}\right)^{2}+24\left(\frac{\psi}{1-\psi}\right)^{3}+24 \frac{q}{1-q}\left(\frac{\psi}{1-\psi}\right)^{2} \\
& \left.+24\left(\frac{q}{1-q}\right)^{2} \frac{\psi}{1-\psi}+24\left(\frac{q}{1-q}\right)^{3}+14 \frac{\psi}{1-\psi}\right) \text {, } \\
& { }_{\alpha} \mu_{2}=\frac{q}{(1-q)^{2}}+\frac{\psi}{(1-\psi)^{2}} \text {, } \\
& { }_{\alpha} \mu_{3}=\frac{q(1+q)}{(1-q)^{3}}+\frac{\psi(1+\psi)}{(1-\psi)^{3}}, \\
& { }_{\alpha} \mu_{4}=\frac{q}{(1-q)^{4}}\left(1+7 q+q^{2}\right)+\frac{\psi}{(1-\psi)^{4}}\left[1+7 \psi+\psi^{2}+\frac{6 q}{(1-q)^{2}}(1-\psi)^{2}\right] \text {, } \\
& V=\frac{\left(q(1-\psi)^{2}+\psi(1-q)^{2}\right)^{1 / 2}}{q(1-\psi)+\psi(1-q)}, \\
& \gamma=\frac{q(1+q)(1-\psi)^{3}+\psi(1-q)^{3}(1+\psi)}{\left(q(1-\psi)^{2}+\psi(1-q)^{2}\right)^{3 / 2}} \text {, }
\end{aligned}
$$




$$
\begin{aligned}
\kappa= & \frac{q\left(1+7 q+q^{2}\right)(1-\psi)^{4}+\psi(1-q)^{4}\left(1+7 \psi+\psi^{2}\right)}{q^{2}(1-\psi)^{4}+2 q \psi(1-q)^{2}(1-\psi)^{2}+\psi^{2}(1-q)^{4}} \\
& +\frac{6 q \psi(1-q)^{2}(1-\psi)^{2}}{q^{2}(1-\psi)^{4}+2 q \psi(1-q)^{2}(1-\psi)^{2}+\psi^{2}(1-q)^{4}}
\end{aligned}
$$

Now we discuss an application of the $\alpha$-modified negative binomial distribution.

ExAmple 1. We study the example given in Panjer and Willmot [PW], p. 211] of automobile insurance claims. They gave results for a sample of 4000 policies and fitted the Poisson distribution (1.3) and the negative binomial distributions (1.2) to the observed data. We extend this study to the $\alpha$ modified negative binomial distribution (2.5) $(X \sim \operatorname{MNB}(\psi, t, \beta /(1+\beta)))$. Our results are presented in Table 1.

Table 1

\begin{tabular}{cccccccc}
\hline \multirow{2}{*}{$\begin{array}{c}\text { Number of } \\
\text { claims }\end{array}$} & Actual & Poisson & \multicolumn{5}{c}{ Theoretical } \\
\cline { 3 - 8 } & & & $\psi=-0.04$ & $\psi=-0.013$ & $\psi=0 ;$ NB & $\psi=0.00005$ & $\psi=0.0008$ \\
\hline 0 & 3719 & 3668.5422 & 3867.9906 & 3767.5716 & 3719.2218 & $\mathbf{3 7 1 9 . 0 3 5 8}$ & 3716.2464 \\
1 & 232 & 317.3289 & 84.3775 & 183.9113 & 229.9011 & $\mathbf{2 3 0 . 0 7 5 5}$ & $\mathbf{2 3 2 . 6 9 0 1}$ \\
2 & 38 & 13.7245 & $\mathbf{3 8 . 1 3 2 0}$ & $\mathbf{3 8 . 0 3 8 7}$ & 39.9107 & 39.9202 & 40.0649 \\
3 & 7 & 0.3957 & $\mathbf{7 . 2 2 7 0}$ & $\mathbf{8 . 0 3 0 5}$ & 8.4156 & 8.4172 & 8.4409 \\
4 & 3 & 0.0086 & 1.7195 & 1.8520 & 1.9313 & $\mathbf{1 . 9 3 1 6}$ & $\mathbf{1 . 9 3 6 5}$ \\
5 & 1 & 0.0001 & 0.4146 & 0.4468 & 0.4648 & $\mathbf{0 . 4 6 4 9}$ & $\mathbf{0 . 4 6 6 0}$ \\
\hline Total & 4000 & 4000 & 3999.8612 & 3999.8510 & 3999.8453 & 3999.8452 & 3999.8449 \\
& $\hat{\lambda}$ & 0.0865 & & & & & \\
& $\hat{t}$ & & 0.216600 & 0.216600 & 0.216600 & 0.216600 & 0.216600 \\
& $\hat{\beta}$ & & 0.399354 & 0.399354 & 0.399354 & 0.399354 & 0.399354 \\
\hline
\end{tabular}

The bold numbers in Table 1 are better fitted to the observed data than the numbers of the negative binomial distribution, i.e. for $\psi=0$; NB means the classical negative binomial distribution.

\begin{tabular}{|c|c|c|c|c|c|}
\hline \multirow{3}{*}{$\begin{array}{c}\text { Empirical } \\
\text { data }\end{array}$} & \multicolumn{5}{|c|}{ Theoretical } \\
\hline & \multirow[t]{2}{*}{ Poisson } & \multicolumn{4}{|c|}{$\alpha$-modified negative binomial } \\
\hline & & $\psi=-0.013$ & $\psi=0 ; \mathrm{NB}$ & $\psi=0.00005$ & 008 \\
\hline $\bar{x}=0.0865$ & $m_{1}=0.0865$ & ${ }_{\alpha} m_{1}=0.0480{ }_{\alpha} m_{1}=0.0737$ & $m_{1}=0.0865$ & ${ }_{\alpha} m_{1}=0.0866$ & $m_{1}=0.0873$ \\
\hline$S^{2}=0.1225$ & $\mu_{2}=0.0865$ & ${ }_{\alpha} \mu_{2}=0.0841 \quad{ }_{\alpha} \mu_{2}=0.1084$ & $\mu_{2}=0.1210$ & ${ }_{\alpha} \mu_{2}=0.1211$ & $\mu_{2}=0.1218$ \\
\hline$\gamma^{\prime}=5.3180$ & $\gamma=3.4001$ & ${ }_{\alpha} \gamma=5.7565$ & $\gamma=5.1700$ & ${ }_{\alpha} \gamma=5.1680$ & ${ }_{\alpha} \gamma=5.1379$ \\
\hline$\kappa^{\prime}=41.0067$ & $\kappa=14.5607$ & ${ }_{\alpha} \kappa=73.4929{ }_{\alpha} \kappa=46.8646$ & $\kappa=38.9623$ & ${ }_{\alpha} \kappa=38.9360$ & ${ }_{\alpha} \kappa=38.5451$ \\
\hline
\end{tabular}

Table 2 
In Table 2 we compare the basic characteristics of the empirical data: first moment $\bar{x}$, variance in a sample $S^{2}$, the coefficient of skewness $\gamma^{\prime}$ and kurtosis $\kappa^{\prime}$ and the theoretical values $\left({ }_{\alpha} m_{1},{ }_{\alpha} \mu_{2},{ }_{\alpha} \gamma,{ }_{\alpha} \kappa\right)$.

3. $\alpha$-Modified Delaporte distribution. The classical Delaporte distribution is a discrete probability distribution commonly applied in actuarial science. It was used to fit the numbers of claims in an automobile insurance portfolio (see [D1, D2, I , [SV, p. 123] and [V, p. 618]).

This distribution can be defined using the convolution of the negative binomial distribution with the Poisson distribution (see [JKK, p. 242]). We see that formula (1.4) can be written in terms of $\alpha(t)$ (see (2.3)) as follows:

$$
P[X=x]=\frac{(\lambda+(\beta /(1+\beta)) \alpha(t))^{x}}{x !(1+\beta)^{t}} e^{-\lambda} .
$$

Chakraborty [C] pointed out that the convolution of a random variable $X$ with the negative binomial distribution $(X \sim \mathrm{NB}(t, \psi))$ and a random variable $Y$ with the Poisson distribution $(Y \sim \mathrm{P}(\lambda))$, where $X$ and $Y$ are independent, is the $\alpha(t)$-modified Poisson distribution $(Z=X+Y \sim$ $\operatorname{MP}(t, \psi, \lambda))$ with the probability function

$$
P[Z=x]=\frac{(\lambda+\psi \alpha(t))^{x}}{x !}(1-\psi)^{t} e^{-\lambda}, \quad x=0,1, \ldots ;
$$

$t>0, \lambda>0$ and $\psi$ are parameters such that $|\psi|<1$ and $\lambda+\psi \geq 0$ and $(\alpha(t))^{l}$ is as in $(2.3)$ (see [C]).

The $\alpha(j)$-modified Poisson distribution is known as the Delaporte distribution (see [C, JKK]). Therefore

$$
\operatorname{Del}(t, \beta, \lambda):=\operatorname{MP}(t, \beta /(1+\beta), \lambda) .
$$

Moreover, it is known that the Delaporte distribution also arises as a mixture of the Poisson distribution $\mathrm{P}(\lambda)$ with the shifted gamma distribution

$$
f(\theta)=\left[\beta^{t} \Gamma(t)\right]^{-1}(\theta-\lambda)^{t-1} e^{-(\theta-\lambda) / \beta}, \quad \theta>\lambda ; \lambda>0, t>0, \beta>0
$$

(see [D1, D2]).

Taking into account the above facts we introduce the $\alpha$-modified Delaporte distribution.

Theorem 3.1. Let $X \sim \operatorname{MNB}(\psi, t, \beta /(1+\beta))$ and $Y \sim \mathrm{P}(\lambda)$. If $X$ and $Y$ are independent random variables and $Z=X+Y$, then

$$
P[Z=z]=(1-\psi) \frac{(\lambda+\psi \alpha(1)+(\beta /(1+\beta)) \alpha(t))^{z}}{z !(1+\beta)^{t}} e^{-\lambda}, \quad z \in \mathbb{N}_{0} ;
$$

$\lambda, \beta>0, t>0, \psi$ are parameters such that $|\psi|<1, \lambda+\psi \geq 0, \psi+\beta /(1+\beta)$ $>0$, and $(\alpha(t))^{l}$ is as in 2.3 . 
Proof. For $X$ and $Y$ as in the statement, the convolution

$$
\begin{aligned}
P[Z=z] & =\sum_{i=0}^{z} P[X=i] P[Y=z-i] \\
& =(1-\psi)(1+\beta)^{-t} e^{-\lambda} \sum_{i=0}^{z} \frac{\left(\psi \alpha(1)+\beta(1+\beta)^{-1} \alpha(t)\right)^{i} \lambda^{z-i}}{i !(z-i) !}
\end{aligned}
$$

is given by $(3.3)$.

Theorem 3.2. Let $Y$ have the $\alpha$-modified Poisson distribution $(Y \sim$ $\operatorname{MP}(\psi, \theta)$ ) as in (2.2). Suppose that $\Theta=\theta$ is the shifted-gamma distributed function (3.2). Then the probability function of $X$ is given by

$$
\begin{aligned}
P[X=x] & =E[P[Y=x \mid \Lambda=\lambda]] \\
& =(1-\psi) \sum_{k=0}^{x} \psi^{x-k} \sum_{i=0}^{k} \frac{\Gamma(t+i) \beta^{i} \lambda^{k-i}}{\Gamma(t) i !(1+\beta)^{t+i}(k-i) !} e^{-\lambda}, \\
& x=0,1,2, \ldots ;
\end{aligned}
$$

$t, \lambda, \beta>0,|\psi|<1$ are the parameters.

Proof. To give the probability function of that mixture we start with

$$
\begin{aligned}
P[X=x] & =\int_{\lambda}^{\infty} \frac{(\theta+\alpha \psi)^{x}}{x !}(1-\psi) e^{-\theta}\left[\beta^{t} \Gamma(t)\right]^{-1}(\theta-\lambda)^{t-1} e^{-(\theta-\lambda) / \beta} d \theta \\
& =\left[\beta^{t} \Gamma(t)\right]^{-1}(1-\psi) e^{-\lambda} \int_{0}^{\infty} \frac{(\lambda+v+\alpha \psi)^{x}}{x !} v^{t-1} e^{-v(\beta+1) / \beta} d v .
\end{aligned}
$$

Hence we get

$$
\begin{aligned}
P[X & =x] \\
& =\frac{(1-\psi) e^{-\lambda}}{\beta^{t} \Gamma(t) x !} \sum_{k=0}^{x}\left(\begin{array}{l}
x \\
k
\end{array}\right)(x-k) ! \psi^{x-k} \sum_{i=0}^{k}\left(\begin{array}{c}
k \\
i
\end{array}\right) \lambda^{k-i} \int_{0}^{\infty} v^{i+t-1} e^{-v(\beta+1) / \beta} d v \\
& =\frac{(1-\psi) e^{-\lambda}}{(1+\beta)^{t}} \sum_{k=0}^{x} \frac{\psi^{x-k}}{k !} \sum_{i=0}^{k}\left(\begin{array}{c}
k \\
i
\end{array}\right) \lambda^{k-i}\left(\frac{\beta}{1+\beta}\right)^{i} \frac{\Gamma(i+t)}{\Gamma(t)} \\
& =(1-\psi) \sum_{k=0}^{x} \psi^{x-k} \sum_{i=0}^{k} \frac{\Gamma(t+i) \beta^{i} \lambda^{k-i}}{\Gamma(t) i !(1+\beta)^{t+i}(k-i) !} e^{-\lambda} .
\end{aligned}
$$

We see that (3.4) can also be written in terms of $\alpha(t)$ as in (3.3) so we have

$$
\begin{aligned}
P[X=x] & =(1-\psi) \frac{(\lambda+\psi \alpha(1)+(\beta /(1+\beta)) \alpha(t))^{x}}{x !(1+\beta)^{t}} e^{-\lambda} \\
& =(1-\psi) \sum_{k=0}^{x} \psi^{x-k} \sum_{i=0}^{k} \frac{\Gamma(t+i) \beta^{i} \lambda^{k-i}}{\Gamma(t) i !(1+\beta)^{t+i}(k-i) !} e^{-\lambda} .
\end{aligned}
$$


Definition 3.3. We call the probability function given by (3.5) the $\alpha$-modified Delaporte distribution $(X \sim \operatorname{MDel}(\psi, t, \beta, \lambda))$ or the generalised Delaporte distribution.

Remark 3.4. We can see that $\operatorname{MDel}(\psi, t, \beta, \lambda)$ with $\psi=0$ is the classical Delaporte distribution in the form (3.1) or (1.4).

Now we give the formulae for $\operatorname{pgf}$ and moments of the $\alpha$-modified Delaporte distribution.

THEOREM 3.5. The probability generating function, the rth factorial moment, the rth moment and the rth central moment of the $\alpha$-modified Delaporte distribution are as follows:

$$
\begin{aligned}
{ }_{\alpha} G_{X}(s) & =(1+\beta)^{-t}(1-\psi) e^{-\lambda} e^{s\left(\psi \alpha(1)+\lambda+\beta(1+\beta)^{-1} \alpha(t)\right)} \\
{ }_{\alpha} m_{(r)} & =\left(\lambda+\alpha(1) \frac{\psi}{1-\psi}+\beta \alpha(t)\right)^{r} \\
& =\sum_{k=0}^{r}\left(\begin{array}{l}
r \\
k
\end{array}\right) t^{[r-k]} \beta^{r-k} \sum_{m=0}^{k} \frac{k !}{(m-k) !}\left(\frac{\psi}{1-\psi}\right)^{m} \lambda^{k-m}, \\
{ }_{\alpha} m_{r} & =\sum_{n=0}^{r} S(r, n)\left(\lambda+\alpha(1) \frac{\psi}{1-\psi}+\beta \alpha(t)\right)^{n} \\
& =\sum_{n=0}^{r} S(r, n) \sum_{k=0}^{n}\left(\begin{array}{l}
n \\
k
\end{array}\right) t^{[n-k]} \beta^{n-k} \sum_{m=0}^{k} \frac{k !}{(m-k) !}\left(\frac{\psi}{1-\psi}\right)^{m} \lambda^{k-m},
\end{aligned}
$$

and

$$
\begin{aligned}
& \alpha \mu_{r}= \sum_{l=0}^{r}\left(\begin{array}{l}
r \\
l
\end{array}\right)(-1)^{r-l}\left({ }_{\alpha} m_{1}\right)^{r-l} \sum_{n=0}^{l} S(l, n)\left(\lambda+\alpha(1) \frac{\psi}{1-\psi}+\beta \alpha(t)\right)^{n} \\
&=\sum_{l=0}^{r}\left(\begin{array}{l}
r \\
l
\end{array}\right)(-1)^{r-l}\left({ }_{\alpha} m_{1}\right)^{r-l} \sum_{n=0}^{l} S(l, n) \sum_{k=0}^{n}\left(\begin{array}{l}
n \\
k
\end{array}\right) t^{[n-k]} \beta^{n-k} \\
& \quad \times \sum_{m=0}^{k} \frac{k !}{(m-k) !}\left(\frac{\psi}{1-\psi}\right)^{m} \lambda^{k-m},
\end{aligned}
$$

where ${ }_{\alpha} m_{1}={ }_{\alpha} E X=\left(\lambda+\alpha(1) \frac{\psi}{1-\psi}+\beta \alpha(t)\right)^{1}=\lambda+t \beta+\frac{\psi}{1-\psi}$.

Proof. Putting (3.3) in (2.13) yields (3.6), which can be written as follows:

$$
{ }_{\alpha} G_{X}(s)=(1+\beta(1-s))^{-t} \frac{1-\psi}{1-s \psi} e^{-\lambda(1-s)} .
$$

The $r$ th derivative of ${ }_{\alpha} G_{X}(s)$ is 


$$
\begin{aligned}
& { }_{\alpha} G_{X}^{(r)}(s) \\
& ={ }_{\alpha} G_{X}(s) \sum_{k=0}^{r}\left(\begin{array}{l}
r \\
k
\end{array}\right) t^{[r-k]}\left(\frac{\beta}{1+\beta(1-s)}\right)^{r-k} \sum_{m=0}^{k} \frac{k !}{(k-m) !} \lambda^{k-m}\left(\frac{\psi}{1-s \psi}\right)^{r} \\
& ={ }_{\alpha} G_{X}(s)\left(\lambda+\alpha(1) \frac{\psi}{1-s \psi}+\alpha(t) \frac{\beta}{1+\beta(1-s)}\right)^{r} .
\end{aligned}
$$

Letting $s=1$ in the above formula we obtain the $r$ th factorial moment of the $\alpha$-modified Delaporte distribution. Using the relations between the moments (2.15) and 2.16) we get the $r$ th moment (3.8) and the $r$ th central moment (3.9) of the $\alpha$-modified Delaporte distribution.

Corollary 3.6. If $X \sim \operatorname{MDel}(\psi, t, \beta, \lambda)$, then

$$
\begin{aligned}
{ }_{\alpha} m_{1}= & \lambda+t \beta+\frac{\psi}{1-\psi}, \\
{ }_{\alpha} m_{2}= & \lambda+\lambda^{2}+t \beta+t^{[2]} \beta^{2}+2 \lambda t \beta+\frac{\psi}{1-\psi}\left(1+2 \lambda+2 t \beta+\frac{2 \psi}{1-\psi}\right), \\
{ }_{\alpha} m_{3}= & \lambda+3 \lambda^{2}+\lambda^{3}+t \beta+3 t^{[2]} \beta^{2}+t^{[3]} \beta^{3}+6 \lambda t \beta+3 \lambda t^{[2]} \beta^{2}+3 \lambda^{2} t \beta \\
& +\frac{\psi}{1-\psi}\left(1+3 \lambda^{2}+3 t^{2} \beta^{[2]}+6 \lambda t \beta+\frac{6 \psi}{(1-\psi)^{2}}+\frac{6 t \beta}{1-\psi}+\frac{6 \lambda}{1-\psi}\right), \\
{ }_{\alpha} m_{4}= & +7 \lambda^{2}+6 \lambda^{3}+\lambda^{4}+t \beta+7 t^{[2]} \beta^{2}+6 t^{[3]} \beta^{3}+t^{[4]} \beta^{4} \\
& +14 \lambda t \beta+18 \lambda t^{[2]} \beta^{2}+4 \lambda t^{[3]} \beta^{3}+18 \lambda^{2} t \beta+6 \lambda^{2} t^{[2]} \beta^{2}+4 \lambda^{3} t \beta \\
& +\frac{\psi}{1-\psi}\left(1+14 \lambda+18 \lambda^{2}+4 \lambda^{3}+14 t \beta+18 t^{[2]} \beta^{2}+4 t^{[3]} \beta^{3}\right. \\
& +12 \lambda^{2} t \beta+14 \frac{\psi}{1-\psi}+36 \lambda \frac{\psi}{1-\psi}+36\left(\frac{\psi}{1-\psi}\right)^{2}+12 \lambda^{2}\left(\frac{\psi}{1-\psi}\right)^{2} \\
& \left.+\left(\lambda+\frac{\psi}{1-\psi}\right)\left(36 t \beta+12 t^{[2]} \beta^{2}+24 t \beta \frac{\psi}{1-\psi}+24\left(\frac{\psi}{1-\psi}\right)^{2}\right)\right) \\
{ }_{\alpha} \mu_{2}= & \lambda+t \beta^{[2]}+\frac{\psi}{(1-\psi)^{2}}, \\
{ }_{\alpha} \mu_{3}= & \lambda+t \beta\left(1+3 \beta+2 \beta^{2}\right)+\frac{\psi}{1-\psi}\left(1+3 \frac{\psi}{1-\psi}+2\left(\frac{\psi}{1-\psi}\right)^{2}\right), \\
{ }_{\alpha} \mu_{4}= & \lambda+3 \lambda^{2}+t \beta\left(1+6 \lambda+6 \lambda \beta+7 \beta+12 \beta^{2}+6 \beta^{3}+3 t \beta+6 t \beta^{2}+3 t \beta^{3}\right) \\
& +\frac{\psi}{1-\psi}\left(1+6 \lambda+6 t \beta^{[2]}\right)+\left(\frac{\psi}{1-\psi}\right)^{2}\left(10+6 \lambda+6 t \beta^{[2]}\right) \\
& +18\left(\frac{\psi}{1-\psi}\right)^{3}+9\left(\frac{\psi}{1-\psi}\right)^{4},
\end{aligned}
$$


and

$$
\begin{aligned}
V & =\frac{\left(\lambda+t \beta^{[2]}+\psi\left(1-2 \lambda-2 t \beta^{[2]}\right)+\psi^{2}\left(\lambda+t \beta^{[2]}\right)\right)^{1 / 2}}{\lambda+t \beta+\psi(1-\lambda-t \beta)}, \\
\gamma & ={ }_{\alpha} \mu_{3} /\left({ }_{\alpha} \mu_{2}\right)^{3 / 2}, \quad \kappa={ }_{\alpha} \mu_{4} /\left({ }_{\alpha} \mu_{2}\right)^{2} .
\end{aligned}
$$

Some of the formulae 3.10 with $\psi=0$ take the forms given in $\mathrm{V}$, p. 619].

Now we present an application of the $\alpha$-modified Delaporte distribution.

ExAmple 2. Delaporte [D2] applied the probability distribution 3.1] to model the number of car accidents. We extend this study to the $\alpha$-modified Delaporte distribution (3.5) $\left(X \sim \operatorname{MDel}\left(\psi, b, 1 / a, s_{0}\right)\right)$. Our results are presented in Table 3.

Table 3

\begin{tabular}{cccccccc}
\hline \multirow{2}{*}{\begin{tabular}{c} 
Number of Number of \\
\cline { 3 - 8 } accidents
\end{tabular}} & cars & Poisson & $\psi=-0.0008$ & $\psi=-0.0005$ & $\psi=0 ;$ Del & $\psi=0.009$ & $\psi=0.01$ \\
\cline { 3 - 8 } & 1316 & 1261.3484 & $\mathbf{1 3 1 6 . 6 1 6 0}$ & $\mathbf{1 3 1 6 . 2 2 1 4}$ & 1315.5636 & 1303.7235 & 1302.4079 \\
1 & 323 & 408.6769 & $\mathbf{3 2 3 . 9 0 5 4}$ & $\mathbf{3 2 4 . 2 0 3 2}$ & 324.6990 & 333.5102 & 334.4760 \\
2 & 81 & 66.2057 & 78.5516 & 78.6250 & 78.7477 & $\mathbf{8 1 . 0 4 0 6}$ & $\mathbf{8 1 . 3 0 5 0}$ \\
3 & 18 & 7.1502 & $\mathbf{1 8 . 9 3 8 1}$ & $\mathbf{1 8 . 9 5 5 9}$ & 18.9858 & 19.5442 & 19.6089 \\
4 & 4 & 0.5762 & $\mathbf{4 . 5 5 2 3}$ & $\mathbf{4 . 5 5 6 6}$ & 4.5638 & 4.6986 & 4.7143 \\
5 & 2 & 0.0375 & 1.0923 & 1.0934 & 1.0951 & $\mathbf{1 . 1 2 7 6}$ & $\mathbf{1 . 1 3 1 4}$ \\
$\geq 6$ & 0 & $\mathbf{0 . 0 0 2 0}$ & 0.2627 & 0.2626 & 0.2625 & $\mathbf{0 . 2 6 0 1}$ & $\mathbf{0 . 2 5 9 9}$ \\
\hline Total & 1744 & 1743.9969 & 1743.9185 & 1743.9181 & 1743.9175 & 1743.9049 & 1743.9035 \\
& $\hat{\lambda}$ & 0.324 & & & & & \\
& $a$ & & 3.1975 & 3.1975 & 3.1975 & 3.1975 & 3.1975 \\
& $b$ & & 1.036 & 1.036 & 1.036 & 1.036 & 1.036 \\
& $s_{0}$ & & 0 & 0 & 0 & 0 & 0 \\
\hline
\end{tabular}

\begin{tabular}{|c|c|c|c|c|c|c|}
\hline \multirow{3}{*}{$\begin{array}{c}\text { Empirical } \\
\text { data }\end{array}$} & \multicolumn{6}{|c|}{ Theoretical } \\
\hline & \multirow[b]{2}{*}{ Poisson } & \multicolumn{5}{|c|}{$\alpha$-modified Delaporte } \\
\hline & & $\psi=-0.0008$ & $\psi=-0.0005$ & $\psi=0 ;$ Del & $\psi=0.009$ & $\psi=0.01$ \\
\hline $\bar{x}=0.3240$ & $m_{1}=0.3240$ & ${ }_{\alpha} m_{1}=0.3232$ & ${ }_{\alpha} m_{1}=0.3235$ & $m_{1}=0.3240$ & ${ }_{\alpha} m_{1}=0.3331$ & ${ }_{\alpha} m_{1}=0.3341$ \\
\hline$S^{2}=0.4243$ & $\mu_{2}=0.3240$ & ${ }_{\alpha} \mu_{2}=0.4245$ & ${ }_{\alpha} \mu_{2}=0.4248$ & $\mu_{2}=0.4253$ & ${ }_{\alpha} \mu_{2}=0.4345$ & ${ }_{\alpha} \mu_{2}=0.4355$ \\
\hline$\gamma^{\prime}=2.4576$ & $\gamma=1.7568$ & $\alpha \gamma=2.4895$ & $\alpha \gamma=2.4906$ & $\gamma=2.4924$ & $\alpha \gamma=2.5260$ & $\alpha \gamma=2.5299$ \\
\hline$\kappa^{\prime}=10.6092$ & $\kappa=6.0864$ & $\alpha \kappa=11.1689$ & ${ }_{\alpha} \kappa=11.1590$ & $\kappa=11.1426$ & ${ }_{\alpha} \kappa=10.8540$ & $\alpha \kappa=10.8226$ \\
\hline
\end{tabular}

Table 4

The bold numbers in Table 3 approximate the number of car accidents better than the classical Delaporte distribution, i.e. for $\psi=0$; Del means Delaporte distribution. 
In Table 4 we give the basic characteristics of the empirical data and the theoretical values.

4. Compound Delaporte distribution with Borel summands. In this section we will be concerned with compound distributions, i.e. distributions of random sums

$$
S_{N}=X_{1}+\cdots+X_{N},
$$

where $\left\{X_{i}, i \geq 1\right\}$ is a sequence of i.i.d. random variables and $N$ is a random variable independent of $\left\{X_{i}, i \geq 1\right\}$.

Finner et al. [FKS] studied the distribution of the random sum 4.1], where $N$ is an independent random variable with the Delaporte distribution and $\left\{X_{i}, i \geq 1\right\}$ is a sequence of independent identically distributed random variables with Borel summands with the probability function

$$
P[X=x]=\frac{(\lambda x)^{x-1}}{x !} e^{-\lambda x}, \quad x=1,2,3, \ldots ; 0<\lambda<1 .
$$

They gave the following theorem.

TheOREM 4.1 (cf. FKS]). Let $\left\{X_{i}, i \geq 1\right\}$ be an i.i.d. sequence with the Borel distribution (4.2) and let $N\left(N \sim \operatorname{Del}\left(m, \frac{\lambda}{1-\lambda}, \theta\right)\right)$ be an independent random variable with the Delaporte distribution (3.1). Then

$$
P\left[S_{N}=n\right]=\frac{(1-\lambda)^{m}(\theta+\lambda n+\lambda \alpha(m-1))^{n}}{n !} e^{-(\theta+\lambda n)}, \quad n \in \mathbb{N}_{0} ;
$$

$\theta \geq 0,0<\lambda<1, m \geq 2$ are parameters, and

$$
E S_{N}=\frac{\theta}{1-\lambda}+\frac{m \lambda}{(1-\lambda)^{2}}, \quad \sigma^{2} S_{N}=\frac{\theta}{(1-\lambda)^{3}}+\frac{m\left(\lambda+\lambda^{2}\right)}{(1-\lambda)^{4}},
$$

where $\alpha^{l}(m-1)=\left(\begin{array}{c}l+m-2 \\ l\end{array}\right) l$ !.

We complete the above investigations by computing the $r$ th moment and the $r$ th central moment of the distribution (4.3), and the coefficients of variation, skewness and kurtosis. To do this we need formulae for the moments of the Borel distribution 4.2 .

We have the following result.

THEOREM 4.2. The rth factorial moment $m_{(r)}$, the rth moment $m_{r}$ and rth central moment $\mu_{r}$ of the Borel distribution (4.2) are as follows:

$$
\begin{aligned}
m_{(r)} & =\frac{\lambda^{r-1}}{(1-\lambda)^{2 r-1}} \sum_{k=0}^{r-1} \lambda^{k} a_{r}(k), \\
m_{r} & =\sum_{n=1}^{r} S(r, n) \frac{\lambda^{n-1}}{(1-\lambda)^{2 n-1}} \sum_{k=0}^{n-1} \lambda^{k} a_{n}(k),
\end{aligned}
$$


$(4.6)$

$$
\mu_{r}=\left(\frac{-1}{1-\lambda}\right)^{r}+\sum_{n=1}^{r}\left(\begin{array}{l}
r \\
n
\end{array}\right)\left(\frac{-1}{1-\lambda}\right)^{r-n} \sum_{k=1}^{n} S(n, k) \frac{\lambda^{k-1}}{(1-\lambda)^{2 k-1}} \sum_{i=0}^{k-1} \lambda^{i} a_{k}(i),
$$

where

$$
a_{n}(k)= \begin{cases}n^{n-1}, & k=0, \\ -(n-1) a_{n-1}(k-1)+(3 n-4-k) a_{n-1}(k)+(k+1) & a_{n-1}(k+1) \\ 0, & 1 \leq k \leq n-1, \\ & k \geq n\end{cases}
$$

Proof. The probability generating function of the Borel distribution 4.2 is given by

$$
G(u)=z, \quad \text { where } \quad z=u e^{\lambda(z-1)}, \quad 0<\lambda<1
$$

(see [CF, p. 158]). By (4.8) we obtain

$$
\frac{d^{r}}{d z^{r}} G(u)=\frac{\lambda^{r-1} e^{r \lambda(z-1)}}{(1-\lambda z)^{2 r-1}} \sum_{k=0}^{r-1} \lambda^{k} a_{r}(k) z^{k},
$$

where $u=z / e^{\lambda(z-1)}$ and $a_{r}(k)$ is given in (4.7). Letting $u=1$ in 4.9) we get the $r$ th factorial moment of the Borel distribution. Using the relations between the moments (2.15) and 2.16) we get the $r$ th moment (4.5) and the $r$ th central moment (4.6) of the Borel distribution.

Some $a_{n}(k)$ are given in Table 5 .

Table 5. The coefficients $a_{n}(k)$

\begin{tabular}{ccccccc}
\hline$n$ & $a_{n}(0)$ & $a_{n}(1)$ & $a_{n}(2)$ & $a_{n}(3)$ & $a_{n}(4)$ & $a_{n}(5)$ \\
\hline 1 & 1 & 0 & 0 & 0 & 0 & 0 \\
2 & 2 & -1 & 0 & 0 & 0 & 0 \\
3 & 9 & -8 & 2 & 0 & 0 & 0 \\
4 & 64 & -79 & 36 & -6 & 0 & 0 \\
5 & 625 & -974 & 622 & -192 & 24 & 0 \\
6 & 7776 & -14543 & 11758 & -5126 & 1200 & -120 \\
\hline
\end{tabular}

Corollary 4.3. If $X$ has the Borel distribution (4.2), then

$$
\begin{aligned}
& m_{(1)}=\frac{1}{1-\lambda}, \quad m_{(2)}=\frac{\lambda(2-\lambda)}{(1-\lambda)^{3}}, \quad m_{(3)}=\frac{\lambda^{2}\left(9-8 \lambda+2 \lambda^{2}\right)}{(1-\lambda)^{5}}, \\
& m_{(4)}=\frac{\lambda^{3}\left(64-79 \lambda+36 \lambda^{2}-6 \lambda^{3}\right)}{(1-\lambda)^{7}}
\end{aligned}
$$


$(4.10)$

$$
\begin{aligned}
m_{1} & =\frac{1}{1-\lambda}, \quad m_{2}=\frac{1}{(1-\lambda)^{3}}, \quad m_{3}=\frac{1+2 \lambda}{(1-\lambda)^{5}}, \quad m_{4}=\frac{1+8 \lambda+6 \lambda^{2}}{(1-\lambda)^{7}}, \\
\mu_{2} & =\frac{\lambda}{(1-\lambda)^{3}}, \quad \mu_{3}=\frac{\lambda(1+2 \lambda)}{(1-\lambda)^{5}}, \\
\mu_{4} & =\frac{\lambda\left(1+11 \lambda+3 \lambda^{2}\right)}{(1-\lambda)^{7}}=\frac{\lambda\left(1+8 \lambda+6 \lambda^{2}\right)}{(1-\lambda)^{7}}+3 \frac{\lambda^{2}}{(1-\lambda)^{6}},
\end{aligned}
$$

and

$$
V=\sqrt{\frac{\lambda}{1-\lambda}}, \quad \gamma=\frac{1+2 \lambda}{\sqrt{\lambda(1-\lambda)}}, \quad \kappa=3+\frac{1+8 \lambda+6 \lambda^{2}}{\lambda(1-\lambda)}
$$

(cf. [CF, pp. 158-159]).

REMARK 4.4. The recurrence relations for the moments $m_{k}$ and for the central moments $\mu_{k}$ of the Borel distribution 4.2 are as follows:

$$
\begin{aligned}
& m_{k+1}=\lambda(1-\lambda)^{-1} \frac{d m_{k}}{d \lambda}+(1-\lambda)^{-1} m_{k}, \quad k=0,1, \ldots, \\
& \mu_{k+1}=\lambda(1-\lambda)^{-1} \frac{d \mu_{k}}{d \lambda}+k \lambda(1-\lambda)^{-3} \mu_{k-1}, \quad k=1,2, \ldots
\end{aligned}
$$

(see [CF, p. 159]).

To give the moments of the compound Delaporte distribution with Borel summands we denote by

- $G_{S_{N}}(s)$ the probability generating function (pgf) of $S_{N}$,

- $E S_{N}^{(r)}$ the $r$ th factorial moment of $S_{N}$,

- $E S_{N}^{r}$ the $r$ th uncorrected moment, for short, the $r$ th moment of $S_{N}$,

- $E\left(S_{N}-E S_{N}\right)^{r}$ the $r$ th central moment of $S_{N}$.

Now we derive formulae for the $r$ th moments of the Delaporte distribution with Borel summands:

THEOREM 4.5. The rth factorial moments, the rth moments and the rth central moments of the compound Delaporte distribution (4.3) with Borel summands are as follows:

$$
\begin{aligned}
& \text { 4.11) } E S_{N}^{(r)}=\left(\theta+\frac{\lambda m}{1-\lambda}\right) m_{(r)}+\sum_{n=2}^{r} \frac{\theta^{n}}{n !} \sum_{j=0}^{n-1}\left(\begin{array}{c}
n \\
j
\end{array}\right)(-1)^{j} G^{(r, n-j)}(1) \\
& +\sum_{k=1}^{r-1}\left(\begin{array}{l}
r \\
k
\end{array}\right) \sum_{i=1}^{k}\left(\frac{\lambda}{1-\lambda}\right)^{i} \frac{m^{[i]}}{i !} \sum_{j=0}^{i-1}\left(\begin{array}{l}
i \\
j
\end{array}\right)(-1)^{j} G^{(k, i-j)}(1) \sum_{n=1}^{r-k} \frac{\theta^{n}}{n !}
\end{aligned}
$$$$
\cdot \sum_{j=0}^{n-1}\left(\begin{array}{c}
n \\
j
\end{array}\right)(-1)^{j} G^{(r-k, n-j)}(1)+\sum_{i=2}^{r}\left(\frac{\lambda}{1-\lambda}\right)^{i} \frac{m^{[i]}}{i !} \sum_{j=0}^{i-1}\left(\begin{array}{l}
i \\
j
\end{array}\right)(-1)^{j} G^{(r, i-j)}(1) \text {, }
$$ 


$$
\begin{gathered}
E S_{N}^{r}=\sum_{k=1}^{r} S(r, k) E S_{N}^{(k)}, \\
E\left(S_{N}-E S_{N}\right)^{r}=\left(-E S_{N}\right)^{r}+\sum_{k=1}^{r}\left(\begin{array}{l}
r \\
k
\end{array}\right) E S_{N}^{k}\left(-E S_{N}\right)^{r-k},
\end{gathered}
$$

where $m_{(r)}$ is the rth factorial moment (4.4) of the Borel distribution,

$$
G^{(r, l)}(s):=\frac{d^{r}}{d s^{r}} G^{l}(s), \quad G^{(r, l)}(1):=\left.\frac{d^{r}}{d s^{r}} G^{l}(s)\right|_{s=1},
$$

and $G(s)$ is the pgf of the Borel distribution (4.8).

Proof. Let $N$ have the Delaporte distribution $\left(N \sim \operatorname{Del}\left(m, \frac{\lambda}{1-\lambda}, \theta\right)\right)$

$$
P[N=n]=(1-\lambda)^{m} \frac{(\theta+\lambda \alpha(m))^{n}}{n !} e^{-\theta}
$$

with the probability generating function

$$
G_{N}(s)=\left(\frac{1-\lambda}{1-\lambda s}\right)^{m} \exp (\theta(s-1))
$$

(see [FKS]), and let $X$ have the Borel distribution (4.2) with pgf $G(s)$. Then taking into account that $G_{S_{N}}(s)=G_{N}(G(s))$ we obtain the pgf of the compound Delaporte distribution with Borel summands:

$$
G_{S_{N}}(s)=\left(\frac{1-\lambda}{1-\lambda G(s)}\right)^{m} \exp (\theta(G(s)-1))
$$

(see [FKS]). Therefore

$$
\begin{aligned}
\frac{d^{r}}{d s^{r}} G_{S_{N}}(s)= & \sum_{k=0}^{r}\left(\begin{array}{l}
r \\
k
\end{array}\right) \frac{d^{k}}{d s^{k}}\left(\frac{1-\lambda}{1-\lambda G(s)}\right)^{m} \frac{d^{r-k}}{d s^{r-k}} \exp (\theta(G(s)-1)) \\
= & \left(\frac{1-\lambda}{1-\lambda G(s)}\right)^{m} \frac{d^{r}}{d s^{r}} \exp (\theta(G(s)-1)) \\
& +\frac{d^{r}}{d s^{r}}\left(\frac{1-\lambda}{1-\lambda G(s)}\right)^{m} \exp (\theta(G(s)-1)) \\
& +\sum_{k=1}^{r-1}\left(\begin{array}{l}
r \\
k
\end{array}\right) \frac{d^{k}}{d s^{k}}\left(\frac{1-\lambda}{1-\lambda G(s)}\right)^{m} \frac{d^{r-k}}{d s^{r-k}} \exp (\theta(G(s)-1)),
\end{aligned}
$$

which gives

$$
\begin{aligned}
\frac{d^{r}}{d s^{r}} G_{S_{N}}(s)= & G_{S_{N}}(s)\left\{\sum_{n=1}^{r} \frac{\theta^{n}}{n !} \sum_{j=0}^{n-1}\left(\begin{array}{c}
n \\
j
\end{array}\right)(-1)^{j} G^{j}(s) G^{(r, n-j)}(s)\right. \\
& +\sum_{i=1}^{r}\left(\frac{\lambda}{1-\lambda s}\right)^{i} \frac{m^{[i]}}{i !} \sum_{j=0}^{i-1}\left(\begin{array}{c}
i \\
j
\end{array}\right)(-1)^{j} G^{j}(s) G^{(r, i-j)}(s)
\end{aligned}
$$




$$
\begin{aligned}
& +\sum_{k=1}^{r-1}\left(\begin{array}{l}
r \\
k
\end{array}\right) \sum_{i=1}^{k}\left(\frac{\lambda}{1-\lambda s}\right)^{i} \frac{m^{[i]}}{i !} \sum_{j=0}^{i-1}\left(\begin{array}{c}
i \\
j
\end{array}\right)(-1)^{j} G^{j}(s) G^{(k, i-j)}(s) \\
& \left.\cdot \sum_{n=1}^{r-k} \frac{\theta^{n}}{n !} \sum_{j=0}^{n-1}\left(\begin{array}{c}
n \\
j
\end{array}\right)(-1)^{j} G^{j}(s) G^{(r-k, n-j)}(s)\right\} .
\end{aligned}
$$

The formula $E S_{N}^{(r)}:=\left.\frac{d^{r}}{d s^{r}} G_{S_{N}}(s)\right|_{s=1}$ leads to 4.11). Taking into account the relations (2.15) and (2.16) between the moments we obtain (4.12) and 4.13 .

The following formulae are known:

$$
\begin{aligned}
& E S_{N}=E N \cdot E X, \quad E\left(S_{N}-E S_{N}\right)=0, \\
& \sigma^{2} S_{N}=E\left(S_{N}-E S_{N}\right)^{2}=E N \sigma^{2} X+\sigma^{2} N E^{2} X, \\
& E\left(S_{N}-E S_{N}\right)^{3}=E N E(X-E X)^{3}+3 \sigma^{2} N E X \sigma^{2} X+E(N-E N)^{3} E^{3} X \\
& (\text { see [KPW, p. 298]), and } \\
& \begin{aligned}
E\left(S_{N}-E S_{N}\right)^{4}= & E N E(X-E X)^{4}+E(N-E N)^{4} E^{4} X \\
& +6 E^{2} X \sigma^{2} X\left[E(N-E N)^{3}+E N \sigma^{2} N\right] \\
& +4 \sigma^{2} N E X E(X-E X)^{3}+3 E N(N-1)\left(\sigma^{2} X\right)^{2}
\end{aligned}
\end{aligned}
$$

(see [SS1], GT]).

Corollary 4.6. If $N \sim \operatorname{Del}\left(m, \frac{\lambda}{1-\lambda}, \theta\right)$ and the i.i.d. summands $\left\{X_{i}\right.$, $i \geq 1\}$ have the Borel distribution 4.2 , then

$$
\begin{aligned}
& E S_{N}=\frac{\theta}{1-\lambda}+\frac{\lambda m}{(1-\lambda)^{2}}, \quad \sigma^{2} S_{N}=\frac{\theta}{(1-\lambda)^{3}}+\frac{\lambda m(1+\lambda)}{(1-\lambda)^{4}}, \\
& E\left(S_{N}-E S_{N}\right)^{3}=\frac{\theta(2 \lambda+1)}{(1-\lambda)^{5}}+\frac{\lambda m\left(2 \lambda^{2}+5 \lambda+1\right)}{(1-\lambda)^{6}}, \\
& E\left(S_{N}-E S_{N}\right)^{4}=\frac{\theta\left(1+8 \lambda+6 \lambda^{2}\right)}{(1-\lambda)^{7}}+\frac{3 \theta^{2}}{(1-\lambda)^{6}}+\frac{6 \theta \lambda m(1+\lambda)}{(1-\lambda)^{7}} \\
& \quad+\frac{\lambda m\left[1+\lambda(3 m+15)+\lambda^{2}(6 m+26)+\lambda^{3}(3 m+6)\right]}{(1-\lambda)^{8}}, \\
& V=\frac{\sqrt{\theta(1-\lambda)+\lambda m(1+\lambda)}}{\theta(1-\lambda)+\lambda m}, \\
& \gamma=\frac{\theta\left(1+\lambda-2 \lambda^{2}\right)+\lambda m\left(1+5 \lambda+2 \lambda^{2}\right)}{(\theta(1-\lambda)+\lambda m(1+\lambda))^{3 / 2}}, \\
& \kappa=\frac{\theta\left(1+7 \lambda-2 \lambda^{2}-6 \lambda^{3}\right)+3 \theta^{2}(1-\lambda)^{2}+6 \theta \lambda m\left(1-\lambda^{2}\right)}{(\theta(1-\lambda)+\lambda m(1+\lambda))^{2}} \\
& +\frac{\lambda m\left[1+\lambda(3 m+15)+\lambda^{2}(6 m+26)+\lambda^{3}(3 m+6)\right]}{(\theta(1-\lambda)+\lambda m(1+\lambda))^{2}} .
\end{aligned}
$$


5. Compound $\alpha$-modified Delaporte distributions. In this section we consider the compound $\alpha$-modified Delaporte distribution, i.e. we study the distribution of the random sum (4.1), where $N \sim \operatorname{MDel}\left(\psi, t, \frac{\lambda}{1-\lambda}, \theta\right)$,

$$
P[N=n]=(1-\lambda)^{t} \frac{(\theta+\psi \alpha(1)+\lambda \alpha(t))^{n}}{n !}(1-\psi) e^{-\theta}, \quad n \in \mathbb{N}_{0},
$$

and $\left\{X_{i}, i \geq 1\right\}$ is a sequence of i.i.d. random variables, where $\alpha(t)$ is defined in 2.3 .

We now extend the concept of the compound Delaporte distribution with Borel summands introduced by Finner et al. [FKS] to the compound $\alpha$-modified Delaporte distribution with Borel summands. To do this we need the following lemma, where we use some ideas of Berg and Nowicki's [BN].

Lemma 5.1. The following equalities hold true:

$$
\begin{aligned}
(\theta+\psi \alpha(1)+\lambda \alpha(t))^{m+1}= & (\theta+\lambda \alpha(t))^{m+1} \\
& +(m+1) \psi(\theta+\psi \alpha(1)+\lambda \alpha(t))^{m}, \\
(\theta+\lambda \alpha(t))^{m+1}= & \lambda t(\theta+\lambda \alpha(t+1))^{m}+\theta(\theta+\lambda \alpha(t))^{m}, \\
\lambda t(\lambda n+\theta+\lambda \alpha(t+1))^{n-1}= & (\lambda n+\theta+\lambda \alpha(t-1))^{n} \\
& -\theta(\lambda n+\theta+\lambda \alpha(t))^{n-1}
\end{aligned}
$$

and

$$
\begin{aligned}
\sum_{m=0}^{n-1}\left(\begin{array}{c}
n-1 \\
m
\end{array}\right) m(\lambda n)^{n-1-m}(\theta+\psi \alpha(1)+\lambda \alpha(t))^{m} & \\
=(n-1)[(\lambda n+\theta & +\psi \alpha(1)+\lambda \alpha(t))^{n-1} \\
& \left.-\lambda n(\lambda n+\theta+\psi \alpha(1)+\lambda \alpha(t))^{n-2}\right] .
\end{aligned}
$$

Proof. From the equality

$$
(\theta+\psi \alpha(1)+\lambda \alpha(t))^{m+1}=\sum_{k=0}^{m+1}\left(\begin{array}{c}
m+1 \\
k
\end{array}\right)(\theta+\lambda \alpha(t))^{k} \psi^{m+1-k} \alpha^{m+1-k},
$$

after some evaluations and using the definition $(2.3)$, we get $(5.2)$.

Note that

$$
\begin{aligned}
& (\theta+\lambda \alpha(t))^{m+1}=\sum_{k=0}^{m+1}\left(\begin{array}{c}
m+1 \\
k
\end{array}\right) \theta^{k} \lambda^{m+1-k} \alpha^{m+1-k}(t) \\
& =\sum_{k=0}^{m}\left(\begin{array}{c}
m \\
k
\end{array}\right) \theta^{k} \lambda^{m+1-k} \alpha^{m+1-k}(t)+\sum_{k=1}^{m+1}\left(\begin{array}{c}
m \\
k-1
\end{array}\right) \theta^{k} \lambda^{m+1-k} \alpha^{m+1-k}(t)
\end{aligned}
$$




$$
\begin{aligned}
& =\lambda t \sum_{k=0}^{m}\left(\begin{array}{c}
m \\
k
\end{array}\right) \theta^{k} \lambda^{m-k} \alpha^{m-k}(t+1)+\theta \sum_{k=0}^{m}\left(\begin{array}{c}
m \\
k
\end{array}\right) \theta^{k} \lambda^{m-k} \alpha^{m-k}(t) \\
& =\lambda t(\theta+\lambda \alpha(t+1))^{m}+\theta(\theta+\lambda \alpha(t))^{m}
\end{aligned}
$$

as $\alpha^{m+1-k}(t)=t \alpha^{m-k}(t+1)$, which ends the proof of (5.3).

Taking into account that

$$
\lambda t(\theta+\lambda \alpha(t+1)+\lambda n)^{n-1}=\lambda^{n} t\left(\frac{\theta}{\lambda}+\alpha(t+1)+n\right)^{n-1}
$$

and using the formula

$$
m(k+\alpha(m+1)+n)^{n-1}=(k+\alpha(m-1)+n)^{n}-k(k+\alpha(m)+n)^{n-1},
$$

we get (5.4).

To prove (5.5) we use the following property of binomial coefficients:

$$
(n-1-m)\left(\begin{array}{c}
n-1 \\
m
\end{array}\right)=(n-1)\left(\begin{array}{c}
n-2 \\
m
\end{array}\right) .
$$

Then

$$
\begin{aligned}
\sum_{m=0}^{n-1}\left(\begin{array}{c}
n-1 \\
m
\end{array}\right) m(\lambda n)^{n-1-m}(\theta+\psi \alpha(1)+\lambda \alpha(t))^{m} \\
=\sum_{m=0}^{n-1}\left(\begin{array}{c}
n-1 \\
m
\end{array}\right)[m-(n-1)+(n-1)](\lambda n)^{n-1-m}(\theta+\psi \alpha(1)+\lambda \alpha(t))^{m} \\
=-\sum_{m=0}^{n-1}\left(\begin{array}{c}
n-1 \\
m
\end{array}\right)(n-1-m)(\lambda n)^{n-1-m}(\theta+\psi \alpha(1)+\lambda \alpha(t))^{m} \\
\quad+(n-1)(\lambda n+\theta+\psi \alpha(1)+\lambda \alpha(t))^{n-1} \\
=-\sum_{m=0}^{n-1}\left(\begin{array}{c}
n-2 \\
m
\end{array}\right)(n-1)(\lambda n)^{n-1-m}(\theta+\psi \alpha(1)+\lambda \alpha(t))^{m} \\
\quad+(n-1)(\lambda n+\theta+\psi \alpha(1)+\lambda \alpha(t))^{n-1} \\
=-\lambda n(n-1)(\lambda n+\theta+\psi \alpha(1)+\lambda \alpha(t))^{n-2} \\
\quad+(n-1)(\lambda n+\theta+\psi \alpha(1)+\lambda \alpha(t))^{n-1},
\end{aligned}
$$

which ends the proof of 5.5 .

The following theorem is a generalisation of the main result of Finner et al. [FKS].

Theorem 5.2. Let $\left\{X_{i}, i \geq 1\right\}$ be an i.i.d. sequence with the Borel distribution (4.2) and let $N$ be an independent random variable with the $\alpha$-modified Delaporte distribution (5.1) $\left(N \sim \operatorname{MDel}\left(\psi, t, \frac{\lambda}{1-\lambda}, \theta\right)\right)$. Then 


$$
\begin{aligned}
& P\left[S_{N}=n\right] \\
= & \frac{(1-\lambda)^{t}(\theta+\lambda n+\psi \alpha(1)+\lambda \alpha(t-1))^{n}}{n !}(1-\psi) e^{-(\theta+\lambda n), \quad n \in \mathbb{N}_{0} ;}
\end{aligned}
$$

$0<\lambda<1, \theta, t>0, \psi$ are parameters such that $|\psi|<1, \lambda+\psi>0$, $\theta+\psi \geq 0$, and $(\alpha(t))^{l}$ is as in 2.3.

Proof. Let $S_{m}=X_{1}+\cdots+X_{m}$. Then $S_{m}$ for each fixed $m \in \mathbb{N}$ has the Borel-Tanner distribution with the probability function

$$
P\left[S_{m}=n\right]=\frac{m(\lambda n)^{n-m}}{n(n-m) !} e^{-\lambda n}, \quad n \in \mathbb{N}, n \geq m
$$

(see [FKS]). Hence using (5.1) and (5.8) we have

$$
\begin{aligned}
& P\left[S_{N}=n\right]=\sum_{m=1}^{\infty} P\left(\sum_{k=1}^{m} X_{k}=n \mid N=m\right) P(N=m) \\
& =\sum_{m=1}^{n} \frac{(\theta+\psi \alpha(1)+\lambda \alpha(t))^{m}}{m !}(1-\lambda)^{t}(1-\psi) e^{-\theta} \frac{m(\lambda n)^{n-m}}{n(n-m) !} e^{-\lambda n} \\
& =\frac{(1-\lambda)^{t}(1-\psi) e^{-(\theta+\lambda n)}}{n !} \sum_{m=0}^{n-1}\left(\begin{array}{c}
n-1 \\
m
\end{array}\right)(\lambda n)^{n-1-m}(\theta+\psi \alpha(1)+\lambda \alpha(t))^{m+1} .
\end{aligned}
$$

Now from 5.2 and 5.3 we obtain

$$
\begin{aligned}
& P\left[S_{N}=n\right] \\
& =\frac{(1-\lambda)^{t}(1-\psi) e^{-(\theta+\lambda n)}}{n !} \sum_{m=0}^{n-1}\left(\begin{array}{c}
n-1 \\
m
\end{array}\right)(\lambda n)^{n-1-m}\left\{(\theta+\lambda \alpha(t))^{m+1}\right. \\
& \left.+(m+1) \psi(\theta+\psi \alpha(1)+\lambda \alpha(t))^{m}\right\} \\
& =\frac{(1-\lambda)^{t}(1-\psi) e^{-(\theta+\lambda n)}}{n !} \sum_{m=0}^{n-1}\left(\begin{array}{c}
n-1 \\
m
\end{array}\right)(\lambda n)^{n-1-m}\left\{\lambda t(\theta+\lambda \alpha(t+1))^{m}\right. \\
& \left.+\theta(\theta+\lambda \alpha(t))^{m}+(m+1) \psi(\theta+\psi \alpha(1)+\lambda \alpha(t))^{m}\right\} \\
& =\frac{(1-\lambda)^{t}(1-\psi) e^{-(\theta+\lambda n)}}{n !}\left\{\lambda t(\lambda n+\theta+\lambda \alpha(t+1))^{n-1}+\theta(\lambda n+\theta+\lambda \alpha(t))^{n-1}\right. \\
& \left.+\sum_{m=0}^{n-1}\left(\begin{array}{c}
n-1 \\
m
\end{array}\right)(\lambda n)^{n-1-m}(m+1) \psi(\theta+\psi \alpha(1)+\lambda \alpha(t))^{m}\right\} .
\end{aligned}
$$

Hence using (5.4) we get

$$
\begin{aligned}
P\left[S_{N}=n\right]= & \frac{(1-\lambda)^{t}(1-\psi) e^{-(\theta+\lambda n)}}{n !}\left\{(\lambda n+\theta+\lambda \alpha(t-1))^{n}\right. \\
& \left.+\sum_{m=0}^{n-1}\left(\begin{array}{c}
n-1 \\
m
\end{array}\right)(\lambda n)^{n-1-m}(m+1) \psi(\theta+\psi \alpha(1)+\lambda \alpha(t))^{m}\right\}
\end{aligned}
$$




$$
\begin{aligned}
= & \frac{(1-\lambda)^{t}(1-\psi) e^{-(\theta+\lambda n)}}{n !}\left\{(\lambda n+\theta+\lambda \alpha(t-1))^{n}\right. \\
& +\psi(\lambda n+\theta+\psi \alpha(1)+\lambda \alpha(t))^{n-1} \\
& \left.+\psi \sum_{m=0}^{n-1}\left(\begin{array}{c}
n-1 \\
m
\end{array}\right)(\lambda n)^{n-1-m} m(\theta+\psi \alpha(1)+\lambda \alpha(t))^{m}\right\} .
\end{aligned}
$$

Therefore by (5.5) we have

$$
\begin{aligned}
P\left[S_{N}=n\right]= & \frac{(1-\lambda)^{t}(1-\psi) e^{-(\theta+\lambda n)}}{n !}\left\{(\lambda n+\theta+\lambda \alpha(t-1))^{n}\right. \\
& +\psi n\left[(\lambda n+\theta+\psi \alpha(1)+\lambda \alpha(t))^{n-1}\right. \\
& \left.\left.-(n-1) \lambda(\lambda n+\theta+\psi \alpha(1)+\lambda \alpha(t))^{n-2}\right]\right\} .
\end{aligned}
$$

But

$$
\begin{aligned}
& (\lambda n+\theta+\psi \alpha(1)+\lambda \alpha(t))^{n-1}-(n-1) \lambda(\lambda n+\theta+\psi \alpha(1)+\lambda \alpha(t))^{n-2} \\
& =\sum_{k=0}^{n-1}\left(\begin{array}{c}
n-1 \\
k
\end{array}\right)(\lambda n+\theta+\alpha \psi)^{k} \lambda^{n-1-k} \alpha^{n-1-k}(t) \\
& \quad-\sum_{k=0}^{n-2}\left(\begin{array}{c}
n-1 \\
k
\end{array}\right)(n-1-k)(\lambda n+\theta+\alpha \psi)^{k} \lambda^{n-1-k} \alpha^{n-2-k}(t) \\
& =\sum_{k=0}^{n-2}\left(\begin{array}{c}
n-1 \\
k
\end{array}\right)(\lambda n+\theta+\alpha \psi)^{k} \lambda^{n-1-k}\left[\alpha^{n-1-k}(t)-(n-1-k) \alpha^{n-2-k}(t)\right] \\
& \quad+(\lambda n+\theta+\alpha \psi)^{n-1} .
\end{aligned}
$$

Taking into account that

$$
\alpha^{n-1-k}(t)-(n-1-k) \alpha^{n-2-k}(t)=\alpha^{n-1-k}(t-1),
$$

we obtain

$$
\begin{aligned}
(\lambda n+\theta+\psi \alpha(1) & +\lambda \alpha(t))^{n-1}-(n-1) \lambda(\lambda n+\theta+\psi \alpha(1)+\lambda \alpha(t))^{n-2} \\
= & \sum_{k=0}^{n-1}\left(\begin{array}{c}
n-1 \\
k
\end{array}\right)(\lambda n+\theta+\alpha \psi)^{k} \lambda^{n-1-k} \alpha^{n-1-k}(t-1) \\
= & (\lambda n+\theta+\psi \alpha(1)+\lambda \alpha(t-1))^{n-1} .
\end{aligned}
$$

Inserting (5.10) in $(5.9)$ we get

$$
\begin{aligned}
P\left[S_{N}=n\right]= & \frac{(1-\lambda)^{t}(1-\psi) e^{-(\theta+\lambda n)}}{n !}\left\{(\lambda n+\theta+\lambda \alpha(t-1))^{n}\right. \\
& \left.+\psi n(\lambda n+\theta+\psi \alpha(1)+\lambda \alpha(t-1))^{n-1}\right\} .
\end{aligned}
$$


Now we apply

$$
(\lambda n+\theta+\psi \alpha(1)+\lambda \alpha(t-1))^{n}=\sum_{k=0}^{n}\left(\begin{array}{l}
n \\
k
\end{array}\right)(\theta+\lambda n+\lambda \alpha(t-1))^{k} \alpha^{n-k} \psi^{n-k},
$$

which, by definition (2.3), leads to

$$
\begin{aligned}
(\lambda n+\theta+\psi \alpha(1)+\lambda \alpha(t-1))^{n}= & (\lambda n+\theta+\lambda \alpha(t-1))^{n} \\
& +\psi n(\lambda n+\theta+\psi \alpha(1)+\lambda \alpha(t-1))^{n-1}
\end{aligned}
$$

and gives the result (5.7).

REMARK 5.3. The main result of Finner et al. [FKS] (see 4.3) follows from (5.7) when $\psi=0$ and $t=m$.

For $\alpha$-modified distributions we denote by

- ${ }_{\alpha} G_{S_{N}}(s)$ the probability generating function (pgf) of $S_{N}$,

- ${ }_{\alpha} M_{S_{N}}(s)$ the moment generating function (mgf) of $S_{N}$,

- ${ }_{\alpha} E S_{N}^{(r)}$ the $r$ th factorial moment of $S_{N}$,

- ${ }_{\alpha} E S_{N}^{r}$ the $r$ th uncorrected moment, for short, the $r$ th moment of $S_{N}$,

- ${ }_{\alpha} E\left(S_{N}-E S_{N}\right)^{r+1}$ the $r$ th central moment of $S_{N}$.

The characteristics of the compound $\alpha$-modified Delaporte distribution are given in the following theorem.

THEOREM 5.4. The probability generating function, the rth factorial moments, the rth moments and the rth central moments of the compound $\alpha$ modified Delaporte distribution (5.7) with Borel summands are as follows:

$$
\begin{aligned}
{ }_{\alpha} G_{S_{N}}(s) & =\left(\frac{1-\lambda}{1-\lambda G(s)}\right)^{t} \frac{1-\psi}{1-\psi G(s)} \exp (\theta(G(s)-1)), \\
{ }_{\alpha} E S_{N}^{(r)}= & E S_{N}^{(r)}+\sum_{i=1}^{r}\left(\frac{\psi}{1-\psi}\right)^{i} \sum_{j=0}^{i-1}\left(\begin{array}{l}
i \\
j
\end{array}\right)(-1)^{j} G^{(r, i-j)}(1) \\
+ & \sum_{k=1}^{r-1}\left(\begin{array}{l}
r \\
k
\end{array}\right) E S_{N}^{(k)} \sum_{i=1}^{r-k}\left(\frac{\psi}{1-\psi}\right)^{i} \sum_{j=0}^{i-1}\left(\begin{array}{l}
i \\
j
\end{array}\right)(-1)^{j} G^{(r-k, i-j)}(1),
\end{aligned}
$$

$$
{ }_{\alpha} E S_{N}^{r}=\sum_{k=1}^{r} S(r, k)_{\alpha} E S_{N}^{(k)}
$$

$$
{ }_{\alpha} E\left(S_{N}-E S_{N}\right)^{r}=\left(-{ }_{\alpha} E S_{N}\right)^{r}+\sum_{k=1}^{r}\left(\begin{array}{l}
r \\
k
\end{array}\right){ }_{\alpha} E S_{N}^{k}\left(-{ }_{\alpha} E S_{N}\right)^{r-k}
$$

where $E S_{N}^{(r)}$ is the rth factorial moment of the compound Delaporte distribution with Borel summands as in 4.11) with $N \sim \operatorname{Del}\left(t, \frac{\lambda}{1-\lambda}, \theta\right)$, 


$$
G^{(r, l)}(s)=\frac{d^{r}}{d s^{r}} G^{l}(s), \quad G^{(r, l)}(1)=\left.\frac{d^{r}}{d s^{r}} G^{l}(s)\right|_{s=1},
$$

and $G(s)$ is the pgf of the Borel distribution (4.8).

Proof. Let $N$ have the $\alpha$-modified Delaporte distribution (5.1) with the pgf

$$
{ }_{\alpha} G_{N}(s)=\left(\frac{1-\lambda}{1-\lambda s}\right)^{t} \frac{1-\psi}{1-\psi s} \exp (\theta(s-1))
$$

and let $X$ have the Borel distribution (4.2) with $G(s)$ in 4.8$)$. Then by the formula ${ }_{\alpha} G_{S_{N}}(s)={ }_{\alpha} G_{N}(G(s))$ we have

$$
{ }_{\alpha} G_{S_{N}}(s)=\left(\frac{1-\lambda}{1-\lambda G(s)}\right)^{t} \frac{1-\psi}{1-\psi G(s)} \exp (\theta(G(s)-1)),
$$

which ends the proof of (5.11). To prove $(5.12)$ we note that

$$
{ }_{\alpha} G_{S_{N}}(s)=G_{S_{N}}(s) \frac{1-\psi}{1-\psi G(s)},
$$

where $G_{S_{N}}(s)$ is as in 4.15 with parameter $t$ instead of $m$, i.e., it is the pgf of the compound Delaporte distribution with Borel summands. Then

$$
\begin{aligned}
\frac{d^{r}}{d s^{r}}{ }_{\alpha} G_{S_{N}}(s)= & \sum_{k=0}^{r}\left(\begin{array}{l}
r \\
k
\end{array}\right) \frac{d^{k}}{d s^{k}} G_{S_{N}}(s) \frac{d^{r-k}}{d s^{r-k}}\left(\frac{1-\psi}{1-\psi G(s)}\right) \\
= & G_{S_{N}}(s) \frac{d^{r}}{d s^{r}}\left(\frac{1-\psi}{1-\psi G(s)}\right)+\left(\frac{1-\psi}{1-\psi G(s)}\right) \frac{d^{r}}{d s^{r}} G_{S_{N}}(s) \\
& +\sum_{k=1}^{r-1}\left(\begin{array}{l}
r \\
k
\end{array}\right) \frac{d^{k}}{d s^{k}} G_{S_{N}}(s) \frac{d^{r-k}}{d s^{r-k}}\left(\frac{1-\psi}{1-\psi G(s)}\right) .
\end{aligned}
$$

The formula ${ }_{\alpha} E S_{N}^{(r)}=\left.\frac{d^{r}}{d s^{r}} \alpha G_{S_{N}}(s)\right|_{s=1}$ ends the proof of $(5.12)$. Taking into account the relations (2.15) and (2.16) we obtain (5.13) and 5.14).

Corollary 5.5. If $N \sim \operatorname{MDel}\left(\psi, t, \frac{\lambda}{1-\lambda}, \theta\right)$ and the i.i.d. summands $\left\{X_{i}, i \geq 1\right\}$ have the Borel distribution 4.2 , then

$$
\begin{aligned}
&{ }_{\alpha} E S_{N}=\frac{\theta}{1-\lambda}+\frac{t \lambda}{(1-\lambda)^{2}}+\frac{\psi}{1-\psi} \cdot \frac{1}{1-\lambda}, \\
&{ }_{\alpha} \sigma^{2} S_{N}={ }_{\alpha} E\left(S_{N}-E S_{N}\right)^{2} \\
&=\frac{\theta}{(1-\lambda)^{3}}+\frac{\lambda t(1+\lambda)}{(1-\lambda)^{4}}+\frac{\psi}{(1-\psi)^{2}} \cdot \frac{1-\lambda \psi}{(1-\lambda)^{3}}, \\
&{ }_{\alpha} E\left(S_{N}-E S_{N}\right)^{3}=\frac{\theta(2 \lambda+1)}{(1-\lambda)^{5}}+\frac{\lambda t\left(2 \lambda^{2}+5 \lambda+1\right)}{(1-\lambda)^{6}} \\
&+\frac{\psi}{1-\psi}\left\{\frac{2 \lambda+1}{(1-\lambda)^{5}}+\frac{3}{(1-\lambda)^{4}} \frac{\psi}{1-\psi}+\frac{2}{(1-\lambda)^{3}}\left(\frac{\psi}{1-\psi}\right)^{2}\right\},
\end{aligned}
$$




$$
\begin{aligned}
&{ }_{\alpha} E\left(S_{N}-E S_{N}\right)^{4}= \frac{\theta\left(1+8 \lambda+6 \lambda^{2}\right)}{(1-\lambda)^{7}}+\frac{3 \theta^{2}}{(1-\lambda)^{6}}+\frac{6 \theta \lambda t(1+\lambda)}{(1-\lambda)^{7}} \\
&+\frac{\lambda t\left(1+\lambda(3 t+15)+\lambda^{2}(6 t+26)+\lambda^{3}(3 t+6)\right)}{(1-\lambda)^{8}} \\
&+\frac{\psi}{1-\psi}\left\{\frac{1+8 \lambda+6 \lambda^{2}}{(1-\lambda)^{7}}+\frac{6 \lambda^{[2]} t}{(1-\lambda)^{7}}+\frac{6 \theta}{(1-\lambda)^{6}}\right\} \\
&+\left(\frac{\psi}{1-\psi}\right)^{2}\left\{\frac{10+8 \lambda}{(1-\lambda)^{6}}+\frac{6 \lambda^{[2]} t}{(1-\lambda)^{6}}+\frac{6 \theta}{(1-\lambda)^{5}}\right\} \\
&+\left(\frac{\psi}{1-\psi}\right)^{3} \cdot \frac{18}{(1-\lambda)^{5}}+\left(\frac{\psi}{1-\psi}\right)^{4} \cdot \frac{9}{(1-\lambda)^{4}}, \\
& V=(\theta(1-\lambda)+\lambda t+\psi\left[(1-\lambda)^{(1-\theta)-\lambda t])^{-1}},\right. \\
& \cdot\left(\theta(1-\lambda)+\lambda t(1+\lambda)+\psi[(1-\lambda)(1-2 \theta)-2 \lambda t]+\psi{ }^{2}(1-\lambda)(\theta-\lambda(1+t))\right)^{1 / 2}, \\
& \gamma={ }_{\alpha} E\left(S_{N}-E S_{N}\right)^{3} /\left({ }_{\alpha} \sigma^{2} S_{N}\right)^{3 / 2}, \\
& \kappa={ }_{\alpha} E\left(S_{N}-E S_{N}\right)^{4} /\left({ }_{\alpha} \sigma^{2} S_{N}\right)^{2} .
\end{aligned}
$$

REMARK 5.6. If $\psi=0$ and $t=m$ in 5.15 , then we get the case studied by Finner et al. [FKS].

Now we focus on the compound $\alpha$-modified Delaporte distribution with gamma summands with the probability density function

$$
f(x)=\frac{\theta^{\rho}}{\Gamma(\rho)} x^{\rho-1} e^{-\theta x}, \quad x>0 ; \theta>0, \rho>0 .
$$

We should mention that the compound Poisson distribution with gamma summands was studied by Withers and Nadarajah [WN]. Some generalisation of their result was presented in SS2] where the compound $\alpha(t)$-modified Poisson distribution with gamma summands was discussed.

We have the following result.

THEOREM 5.7. The moment generating function, the rth moments and the $(r+1)$ th central moments of the compound $\alpha$-modified Delaporte distribution with gamma summands are as follows:

$$
\begin{aligned}
{ }_{\alpha} M_{S_{N}}(s)= & \exp \left\{-\lambda\left[1-\left(1-\frac{s}{\theta}\right)^{-\rho}\right]\right\} \\
\cdot & {\left[1+\beta\left(1-\left(1-\frac{s}{\theta}\right)^{-\rho}\right)\right]^{-t} \frac{1-\psi}{1-\psi(1-s / \theta)^{-\rho}}, }
\end{aligned}
$$

$$
{ }_{\alpha} E S_{N}^{r}=\theta^{-r} \sum_{k=1}^{r}\left(\lambda+\beta \alpha(t)+\alpha \frac{\psi}{1-\psi}\right)^{k} \sum_{i=0}^{k-1}(-1)^{i} \frac{1}{i !(k-i) !}(\rho(k-i))^{[r]},
$$




$$
\begin{aligned}
= & \theta^{-r-1} \sum_{l=1}^{r}\left(\begin{array}{c}
r \\
l
\end{array}\right)\left[-\rho\left(\lambda+\beta t+\frac{\psi}{1-\psi}\right)\right]^{r-l}\left\{\sum_{k=1}^{l}\left(\lambda+\beta \alpha(t)+\alpha \frac{\psi}{1-\psi}\right)^{k}\right. \\
& \cdot \sum_{i=0}^{k-1}(-1)^{i} \frac{\rho\left(k-i-\lambda-\frac{\psi}{1-\psi}-\beta t\right)+l}{i !(k-i) !}(\rho(k-i))^{[l]} \\
& \left.+\left(\lambda+\beta \alpha(t)+\alpha \frac{\psi}{1-\psi}\right)^{l+1} \sum_{i=0}^{l}(-1)^{i} \frac{\rho(l+1-i)+l}{i !(l+1-i) !}(\rho(l+1-i))^{[l]}\right\} .
\end{aligned}
$$

Proof. It is known that the moment generating function of $S_{N}$ satisfies

$$
M_{S_{N}}(s)=G_{N}\left(M_{X}(s)\right),
$$

where $M_{X}(s)$ denotes the mgf of $X$. Inserting the pgf of the $\alpha$-modified Delaporte distribution,

$$
{ }_{\alpha} G_{N}(s)=(1+\beta(1-s))^{-t} \exp \{-\lambda(1-s)\} \frac{1-\psi}{1-\psi s},
$$

and the mgf of the gamma distribution, $M_{X}(s)=(1-s / \theta)^{-\rho}$, in 5.20 , we obtain (5.17).

The $r$ th derivative of ${ }_{\alpha} M_{S_{N}}(s)$ in (5.17) is given by

$$
\begin{aligned}
\frac{d^{r}}{d s^{r}}{ }_{\alpha} M_{S_{N}}(s) & ={ }_{\alpha} M_{S_{N}}(s) \theta^{-r} \sum_{k=1}^{r} \frac{1}{k !}\left(1-\frac{s}{\theta}\right)^{-\rho k-r} \sum_{i=0}^{k}\left(\begin{array}{c}
k \\
i
\end{array}\right) \\
& \cdot t^{[k-i]}\left(\frac{\beta}{1+\beta\left[1-(1-s / \theta)^{-\rho}\right]}\right)^{k-i} \sum_{m=0}^{i} \frac{i !}{(i-m) !} \lambda^{i-m} \\
& \cdot\left(\frac{\psi}{1-\psi(1-s / \theta)^{-\rho}}\right)^{m} \sum_{l=0}^{k-1}\left(\begin{array}{l}
k \\
l
\end{array}\right)(-1)^{l}(\rho(k-l))^{[r]}
\end{aligned}
$$

Letting $s=0$ in (5.21) we obtain (5.18). Using the relation

$$
{ }_{\alpha} E\left(S_{N}-E S_{N}\right)^{r}=\sum_{l=0}^{r}\left(\begin{array}{l}
r \\
l
\end{array}\right)\left(-{ }_{\alpha} E S_{N}\right)^{r-l}{ }_{\alpha} E S_{N}^{l}
$$

we get

$$
{ }_{\alpha} E\left(S_{N}-E S_{N}\right)^{r+1}=\left(-{ }_{\alpha} E S_{N}\right)^{r+1}+\sum_{l=1}^{r+1}\left(\begin{array}{c}
r+1 \\
l
\end{array}\right)\left(-{ }_{\alpha} E S_{N}\right)^{r+1-l}{ }_{\alpha} E S_{N}^{l} .
$$

Therefore 


$$
\begin{aligned}
&{ }_{\alpha} E\left(S_{N}-E S_{N}\right)^{r+1} \\
&=\left(-{ }_{\alpha} E S_{N}\right)^{r+1}+\sum_{l=1}^{r}\left(\begin{array}{c}
r \\
l
\end{array}\right)\left({ }_{\alpha} E S_{N}\right)^{r+1-l}{ }_{\alpha} E S_{N}^{l} \\
&+\sum_{l=1}^{r+1}\left(\begin{array}{c}
r \\
l-1
\end{array}\right)\left(-{ }_{\alpha} E S_{N}\right)^{r+1-l}{ }_{\alpha} E S_{N}^{l} \\
&= \sum_{l=1}^{r}\left(\begin{array}{l}
r \\
l
\end{array}\right)\left(-{ }_{\alpha} E S_{N}\right)^{r-l}\left[-\left({ }_{\alpha} E S_{N}\right)_{\alpha} E S_{N}^{l}+{ }_{\alpha} E S_{N}^{l+1}\right] .
\end{aligned}
$$

Taking into account 5.22 and $(5.18)$ we obtain

$$
\begin{aligned}
{ }_{\alpha} E\left(S_{N}-E S_{N}\right)^{r} & \theta^{-r-1} \sum_{l=1}^{r}\left(\begin{array}{l}
r \\
l
\end{array}\right)\left[-\rho\left(\lambda+\beta t+\frac{\psi}{1-\psi}\right)\right]^{r-l}\left\{-\rho\left(\lambda+\beta t+\frac{\psi}{1-\psi}\right)\right. \\
& \cdot \sum_{k=1}^{l}\left(\lambda+\beta \alpha(t)+\alpha \frac{\psi}{1-\psi}\right)^{k} \sum_{i=0}^{k-1}(-1)^{i} \frac{1}{i !(k-i) !}(\rho(k-i))^{[l]} \\
& +\sum_{k=1}^{l}\left(\lambda+\beta \alpha(t)+\alpha \frac{\psi}{1-\psi}\right)^{k} \sum_{i=0}^{k-1}(-1)^{i} \frac{\rho(k-i)+l}{i !(k-i) !}(\rho(k-i))^{[l]} \\
& \left.+\left(\lambda+\beta \alpha(t)+\alpha \frac{\psi}{1-\psi}\right)^{l+1} \sum_{i=0}^{l}(-1)^{i} \frac{\rho(l+1-i)+l}{i !(l+1-i) !}(\rho(l+1-i))^{[l]}\right\} .
\end{aligned}
$$

Hence we get

$$
\begin{aligned}
{ }_{\alpha} E & \left(S_{N}-E S_{N}\right)^{r+1} \\
= & \theta^{-r-1} \sum_{l=1}^{r}\left(\begin{array}{l}
r \\
l
\end{array}\right)\left[-\rho\left(\lambda+\beta t+\frac{\psi}{1-\psi}\right)\right]^{r-l}\left\{\sum_{k=1}^{l}\left(\lambda+\beta \alpha(t)+\alpha \frac{\psi}{1-\psi}\right)^{k}\right. \\
& \cdot \sum_{i=0}^{k-1}(-1)^{i} \frac{\rho(k-i)+l-\rho\left(\lambda+\beta t+\frac{\psi}{1-\psi}\right)}{i !(k-i) !}(\rho(k-i))^{[l]} \\
& \left.+\left(\lambda+\beta \alpha(t)+\alpha \frac{\psi}{1-\psi}\right)^{l+1} \sum_{i=0}^{l}(-1)^{i} \frac{\rho(l+1-i)+l}{i !(l+1-i) !}(\rho(l+1-i))^{[l]}\right\}
\end{aligned}
$$

which ends the proof.

REMARK 5.8. For $\psi=0$ see [SS2], and for $\psi=0, \beta=0$ see [WN].

Corollary 5.9. If $N \sim \operatorname{MDel}(\psi, t, \beta, \lambda)$ and the i.i.d. summands $\left\{X_{i}\right.$, $i \geq 1\}$ have the gamma distribution, then 


$$
\begin{aligned}
& { }_{\alpha} E S_{N}=\frac{\rho}{\theta}\left(\lambda+t \beta+\frac{\psi}{1-\psi}\right) \\
& { }_{\alpha} E S_{N}^{2}=\frac{\rho}{\theta^{2}}\left(\lambda(\rho+1)+\lambda^{2} \rho+t \beta[\rho+1+2 \lambda \rho]+t^{[2]} \beta^{2} \rho\right. \\
& \left.+\frac{\psi}{1-\psi}\left[\rho+1+2 \lambda \rho+2 \frac{\psi}{1-\psi} \rho+2 \beta t \rho\right]\right) \\
& { }_{\alpha} E S_{N}^{3}=\frac{\rho}{\theta^{3}}\left(\lambda(\rho+1)^{[2]}+3 \lambda^{2} \rho^{[2]}+\lambda^{3} \rho^{2}+t \beta\left[(\rho+1)^{[2]}+6 \lambda \rho^{[2]}+3 \lambda^{2} \rho^{2}\right]\right. \\
& +t^{[2]} \beta^{2}\left[3 \rho^{[2]}+3 \lambda \rho^{2}\right]+t^{[3]} \beta^{3} \rho^{2} \\
& +\frac{\psi}{1-\psi}\left[(\rho+1)^{[2]}+6 \lambda \rho^{[2]}+3 \lambda^{2} \rho^{2}+3 \beta^{2} t^{[2]} \rho^{2}+6 \beta t\left(\rho^{[2]}+\lambda \rho^{2}\right)\right] \\
& \left.+6\left(\frac{\psi}{1-\psi}\right)^{2}\left[\rho^{[2]}+\lambda \rho^{2}+\beta t \rho^{2}+\frac{\psi}{1-\psi} \rho^{2}\right]\right), \\
& { }_{\alpha} E S_{N}^{4}=\frac{\rho}{\theta^{4}}\left(\lambda(\rho+1)^{[3]}+\lambda^{2}\left[3 \rho^{[2]}(\rho+1)+4 \rho^{[3]}\right]+6 \lambda^{3} \rho \rho^{[2]}+\lambda^{4} \rho^{3}\right. \\
& +\beta t\left((\rho+1)^{[3]}+2 \lambda\left(3 \rho^{[2]}(\rho+1)+4 \rho^{[3]}\right)+18 \lambda^{2} \rho^{[2]} \rho+4 \lambda^{3} \rho^{3}\right) \\
& +\beta^{2} t^{[2]}\left(3 \rho^{[2]}(\rho+1)+4 \rho^{[3]}+18 \lambda \rho^{[2]} \rho+6 \lambda^{2} \rho^{3}\right) \\
& +\beta^{3} t^{[3]}\left(6 \rho^{[2]} \rho+4 \lambda \rho^{[3]}\right)+\beta^{4} t^{[4]} \rho^{3} \\
& +\frac{\psi}{1-\psi}\left[(\rho+1)^{[3]}+2 \lambda\left(3 \rho^{[2]}(\rho+1)+4 \rho^{[3]}\right)+18 \lambda^{2} \rho^{[2]} \rho+4 \lambda^{3} \rho^{3}\right. \\
& +\beta t\left(2\left(3 \rho^{[2]}(\rho+1)+4 \rho^{[3]}\right)+36 \lambda \rho^{[2]} \rho+12 \lambda^{2} \rho^{3}+24 \lambda \frac{\psi}{1-\psi} \rho^{3}\right. \\
& \left.+24\left(\frac{\psi}{1-\psi}\right)^{2} \rho^{3}+36 \frac{\psi}{1-\psi} \rho^{[2]} \rho\right)+\beta^{2} t^{[2]}\left(12 \lambda \rho^{3}+12 \frac{\psi}{1-\psi} \rho^{3}+18 \rho^{[2]} \rho\right) \\
& \left.+4 \beta^{3} t^{[3]} \rho^{3}\right]+\left(\frac{\psi}{1-\psi}\right)^{2}\left[2\left(3 \rho^{[2]}(\rho+1)+4 \rho^{[3]}\right)+36 \lambda \rho^{[2]} \rho+12 \lambda^{2} \rho^{3}\right] \\
& \left.+24 \lambda\left(\frac{\psi}{1-\psi}\right)^{3} \rho^{3}+24\left(\frac{\psi}{1-\psi}\right)^{4} \rho^{3}+36\left(\frac{\psi}{1-\psi}\right)^{2} \rho^{[2]} \rho^{2}\right), \\
& { }_{\alpha} \sigma^{2} S_{N}=\frac{\rho}{\theta^{2}}\left(\lambda(\rho+1)+t \beta(\rho+1+\beta \rho)+\frac{\psi}{1-\psi}\left(1+\frac{\rho}{1-\psi}\right)\right), \\
& { }_{\alpha} E\left(S_{N}-E S_{N}\right)^{3}=\frac{\rho}{\theta^{3}}\left(\lambda(\rho+1)^{[2]}+t \beta\left((\rho+1)^{[2]}+3 \beta \rho^{[2]}+2 \beta^{2} \rho^{2}\right)\right. \\
& \left.+\frac{\psi}{1-\psi}\left[(\rho+1)^{[2]}+3 \frac{\psi}{1-\psi} \rho^{[2]}+2\left(\frac{\psi}{1-\psi}\right)^{2} \rho^{2}\right]\right),
\end{aligned}
$$




$$
\begin{aligned}
&{ }_{\alpha} E\left(S_{N}-E S_{N}\right)^{4}=\frac{\rho}{\theta^{4}}\left(\lambda(\rho+1)^{[3]}+3 \lambda^{2} \rho^{[2]}(\rho+1)+t \beta\left((\rho+1)^{[3]}\right.\right. \\
&\left.+6 \lambda \rho^{[2]}(\rho+1)\right)+t \beta^{2}\left(6 \lambda \rho^{[2]} \rho+3 t \rho^{[2]}(\rho+1)+7 \rho^{[2]} \rho+11 \rho^{[2]}\right) \\
&+6 \beta^{3}\left(t^{[2]}+t\right) \rho^{[2]} \rho+3 \beta^{4}\left(t^{[2]}+t\right) \rho^{3} \\
&+\frac{\psi}{1-\psi}\left[(\rho+1)^{[3]}+6 \lambda \rho^{[2]}(\rho+1)+6 t \beta \rho^{[2]}(\rho+1)+6 t \beta^{2} \rho^{[2]} \rho\right] \\
&+\left(\frac{\psi}{1-\psi}\right)^{2}\left[10 \rho^{[2]} \rho+14 \rho^{[2]}+6 \lambda \rho^{[2]} \rho+6 t \beta \rho^{[2]} \rho+6 t \beta^{2} \rho^{3}\right] \\
&\left.+18\left(\frac{\psi}{1-\psi}\right)^{3} \rho^{[2]} \rho+9\left(\frac{\psi}{1-\psi}\right)^{4} \rho^{3}\right), \\
& V=(\rho[\lambda+t \beta+\psi(1-\lambda-t \beta)])^{-1} \\
& \cdot(\rho[\lambda(\rho+1)+t \beta(\rho+1+\beta \rho)+\psi(1-2 t \beta(\rho+1+\beta \rho)+\rho-2 \lambda(\rho+1)) \\
& \gamma={ }_{\alpha} E\left(S_{N}-E S_{N}\right)^{3} /\left(\alpha \sigma^{2} S_{N}\right)^{3 / 2}, \quad \kappa={ }_{\alpha} E\left(S_{N}-E S_{N}\right)^{4} /\left({ }_{\alpha} \sigma^{2} S_{N}\right)^{2} .
\end{aligned}
$$

In the particular case of $\rho=1$, we get formulae for the compound $\alpha$-modified Delaporte distribution with exponential summands: $N \sim$ $\operatorname{MDel}(\psi, t, \beta, \lambda)$ and $f(x)=\theta e^{-\theta x}$.

THEOREM 5.10. The moment generating function, the rth moments and the $(r+1)$ th central moments of the compound $\alpha$-modified Delaporte distribution with exponential summands are as follows:

$$
\begin{aligned}
& { }_{\alpha} M_{S_{N}}(s) \\
& =\exp \left\{-\lambda\left[1-\left(1-\frac{s}{\theta}\right)^{-1}\right]\right\}\left[1+\beta\left(1-\left(1-\frac{s}{\theta}\right)^{-1}\right)\right]^{-t} \frac{1-\psi}{1-\psi(1-s / \theta)^{-1}}, \\
& \alpha E S_{N}^{r}=\theta^{-r} \sum_{k=1}^{r}\left(\lambda+\beta \alpha(t)+\alpha \frac{\psi}{1-\psi}\right)^{k} \sum_{i=0}^{k-1}(-1)^{i} \frac{1}{i !(k-i) !}(k-i)^{[r]}, \\
& \alpha E\left(S_{N}-E S_{N}\right)^{r+1}=\theta^{-r-1} \sum_{l=1}^{r}\left(\begin{array}{l}
r \\
l
\end{array}\right)\left[-\left(\lambda+\beta t+\frac{\psi}{1-\psi}\right)\right]^{r-l} \\
& \cdot\left\{\sum_{k=1}^{l}\left(\lambda+\beta \alpha(t)+\alpha \frac{\psi}{1-\psi}\right)^{k} \sum_{i=0}^{k-1}(-1)^{i} \frac{k-i-\lambda-\frac{\psi}{1-\psi}-\beta t+l}{i !(k-i) !}(k-i)^{[l]}\right. \\
& \left.+\left(\lambda+\beta \alpha(t)+\alpha \frac{\psi}{1-\psi}\right)^{l+1} \sum_{i=0}^{l}(-1)^{i} \frac{2 l+1-i}{i !(l+1-i) !}(l+1-i)^{[l]}\right\} .
\end{aligned}
$$

Corollary 5.11. If $N \sim \operatorname{MDel}(\psi, t, \beta, \lambda)$ and the i.i.d. summands $\left\{X_{i}, i \geq 1\right\}$ have the exponential distribution, then 


$$
\begin{aligned}
& { }_{\alpha} E S_{N}=\theta^{-1}\left(\lambda+t \beta+\frac{\psi}{1-\psi}\right), \\
& { }_{\alpha} E S_{N}^{2}=\theta^{-2}\left(2 \lambda+\lambda^{2}+2 t \beta(\lambda+1)+t^{[2]} \beta^{2}+2 \frac{\psi}{1-\psi}\left[1+\lambda+\beta t+\frac{\psi}{1-\psi}\right]\right), \\
& { }_{\alpha} E S_{N}^{3}=\theta^{-3}\left(6 \lambda+18 \lambda^{2}+\lambda^{3}+t \beta\left(6+6 \lambda+3 \lambda^{2}\right)+t^{[2]} \beta^{2}(6+3 \lambda)+\beta^{3} t^{[3]}\right. \\
& +\frac{\psi}{1-\psi}\left[6+12 \lambda+3 \lambda^{2}+3 t^{[2]} \beta^{2}+6 \beta t(2+\lambda)\right] \\
& \left.+6\left(\frac{\psi}{1-\psi}\right)^{2}\left(2+\lambda+\beta t+\frac{\psi}{1-\psi}\right)\right) \\
& { }_{\alpha} E S_{N}^{4}=\theta^{-4}\left(24 \lambda+36 \lambda^{2}+12 \lambda^{3}+\lambda^{4}+t \beta\left(24+72 \lambda+36 \lambda^{2}+4 \lambda^{3}\right)\right. \\
& +t^{[2]} \beta^{2}\left(36+36 \lambda+6 \lambda^{2}\right)+t^{[3]} \beta^{3}(4 \lambda+12)+t^{[4]} \beta^{4} \\
& +\frac{\psi}{1-\psi}\left[24+72 \lambda+36 \lambda^{2}+4 \lambda^{3}+t \beta\left(72+72 \lambda+12 \lambda^{2}\right.\right. \\
& \left.+24 \lambda \frac{\psi}{1-\psi}+24\left(\frac{\psi}{1-\psi}\right)^{2}+72 \frac{\psi}{1-\psi}\right) \\
& \left.+t^{[2]} \beta^{2}\left(12 \lambda+12 \frac{\psi}{1-\psi}+36\right)+4 \beta^{3} t^{[3]}\right] \\
& \left.+\left(\frac{\psi}{1-\psi}\right)^{2}\left(72+72 \lambda+12 \lambda^{2}\right)+\left(\frac{\psi}{1-\psi}\right)^{3}(24 \lambda+72)+24\left(\frac{\psi}{1-\psi}\right)^{4}\right) \\
& { }_{\alpha} \sigma^{2} S_{N}=\theta^{-2}\left(2 \lambda+t \beta(2+\beta)+\frac{\psi}{1-\psi}\left(2+\frac{\psi}{1-\psi}\right)\right), \\
& { }_{\alpha} E\left(S_{N}-E S_{N}\right)^{3}=\theta^{-3}\left(6 \lambda+t \beta\left(6+6 \beta+2 \beta^{2}\right)\right. \\
& \left.+\frac{\psi}{1-\psi}\left(6+6 \frac{\psi}{1-\psi}+2\left(\frac{\psi}{1-\psi}\right)^{2}\right)\right) \\
& { }_{\alpha} E\left(S_{N}-E S_{N}\right)^{4}=\theta^{-4}\left(24 \lambda+12 \lambda^{2}+t \beta(24+24 \lambda)\right. \\
& +t \beta^{2}(12 \lambda+12 t+36)+12 \beta^{3}\left(t^{[2]}+t\right)+3 \beta^{4}\left(t^{[2]}+t\right) \\
& +\frac{\psi}{1-\psi}\left[24+24 \lambda+24 t \beta+12 t \beta^{2}\right] \\
& \left.+\left(\frac{\psi}{1-\psi}\right)^{2}\left[48+12 \lambda+12 t \beta+6 t \beta^{2}\right]+36\left(\frac{\psi}{1-\psi}\right)^{3}+9\left(\frac{\psi}{1-\psi}\right)^{4}\right) \\
& V=(\lambda+t \beta+\psi(1-\lambda-t \beta))^{-1}(2 \lambda+t \beta(2+\beta) \\
& \left.+2 \psi(1-2 \lambda-t \beta(2+\beta))+\psi^{2}(2 \lambda+t \beta(2+\beta)-1)\right)^{1 / 2},
\end{aligned}
$$




$$
\gamma={ }_{\alpha} E\left(S_{N}-E S_{N}\right)^{3} /\left({ }_{\alpha} \sigma^{2} S_{N}\right)^{3 / 2}, \quad \kappa={ }_{\alpha} E\left(S_{N}-E S_{N}\right)^{4} /\left({ }_{\alpha} \sigma^{2} S_{N}\right)^{2} .
$$

6. Compound $\alpha$-modified negative binomial distribution with gamma summands. Klugman et al. [KPW, p. 306] considered the compound negative binomial distribution with exponential summands. We study the compound $\alpha$-modified negative binomial distribution with gamma summands, viz. $N \sim \operatorname{MNB}\left(\psi, t, \frac{\beta}{1+\beta}\right)$ and the i.i.d. summands $\left\{X_{i}, i \geq 1\right\}$ have the gamma distribution (5.16).

THEOREM 6.1. The moment generating function, the rth moments and the $(r+1)$ th central moments of the compound $\alpha$-modified negative binomial distribution with gamma summands are as follows:

$$
\begin{aligned}
& { }_{\alpha} M_{S_{N}}(s)=\left[1+\beta\left(1-\left(1-\frac{s}{\theta}\right)^{-\rho}\right)\right]^{-t} \frac{1-\psi}{1-\psi(1-s / \theta)^{-\rho}} \\
& { }_{\alpha} E S_{N}^{r}=\theta^{-r} \sum_{k=1}^{r}\left(\beta \alpha(t)+\alpha \frac{\psi}{1-\psi}\right)^{k} \sum_{i=0}^{k-1}(-1)^{i} \frac{1}{i !(k-i) !}(\rho(k-i))^{[r]} \\
& { }_{\alpha} E\left(S_{N}-E S_{N}\right)^{r+1}=\theta^{-r-1} \sum_{l=1}^{r}\left(\begin{array}{l}
r \\
l
\end{array}\right)\left[-\rho\left(\beta t+\frac{\psi}{1-\psi}\right)\right]^{r-l} \\
& \cdot\left\{\sum_{k=1}^{l}\left(\beta \alpha(t)+\alpha \frac{\psi}{1-\psi}\right)^{k} \sum_{i=0}^{k-1}(-1)^{i} \frac{\rho\left(k-i-\frac{\psi}{1-\psi}-\beta t\right)+l}{i !(k-i) !}\right. \\
& \cdot(\rho(k-i))^{[l]}+\left(\beta \alpha(t)+\alpha \frac{\psi}{1-\psi}\right)^{l+1} \sum_{i=0}^{l}(-1)^{i} \frac{\rho(l+1-i)+l}{i !(l+1-i) !} \\
& \left.\cdot(\rho(l+1-i))^{[l]}\right\} .
\end{aligned}
$$

Proof. Taking the pgf of the $\alpha$-modified negative binomial distribution,

$$
{ }_{\alpha} G_{N}(s)=(1+\beta(1-s))^{-t} \frac{1-\psi}{1-\psi s},
$$

and the mgf of the gamma distribution, $M_{X}(s)=(1-s / \theta)^{-\rho}$, in 5.20) we obtain 6.1).

The $r$ th derivative of ${ }_{\alpha} M_{S_{N}}(s)$ in 6.1) is given by

$$
\begin{aligned}
& \frac{d^{r}}{d s^{r}}{ }_{\alpha} M_{S_{N}}(s)={ }_{\alpha} M_{S_{N}}(s) \theta^{-r} \sum_{k=1}^{r} \frac{1}{k !}\left(1-\frac{s}{\theta}\right)^{-\rho k-r} \sum_{i=0}^{k} k_{(i)} t^{[k-i]} \\
& \cdot\left(\frac{\beta}{1+\beta\left[1-(1-s / \theta)^{-\rho]}\right.}\right)^{k-i}\left(\frac{\psi}{1-\psi(1-s / \theta)^{-\rho}}\right)^{i k-1} \sum_{l=0}^{k}\left(\begin{array}{l}
k \\
l
\end{array}\right)(-1)^{l} \\
& \cdot(\rho(k-l))^{[r]} .
\end{aligned}
$$


Setting $s=0$ in (6.4) we obtain (6.2). Using (5.22) and (6.2) we obtain

$$
\begin{aligned}
& { }_{\alpha} E\left(S_{N}-E S_{N}\right)^{r}=\theta^{-r-1} \sum_{l=1}^{r}\left(\begin{array}{l}
r \\
l
\end{array}\right)\left[-\rho\left(\beta t+\frac{\psi}{1-\psi}\right)\right]^{r-l} \\
& \quad \cdot\left\{-\rho\left(\beta t+\frac{\psi}{1-\psi}\right) \sum_{k=1}^{l}\left(\beta \alpha(t)+\alpha \frac{\psi}{1-\psi}\right)^{k} \sum_{i=0}^{k-1}(-1)^{i} \frac{1}{i !(k-i) !}(\rho(k-i))^{[l]}\right. \\
& \quad+\sum_{k=1}^{l}\left(\beta \alpha(t)+\alpha \frac{\psi}{1-\psi}\right)^{k} \sum_{i=0}^{k-1}(-1)^{i} \frac{\rho(k-i)+l}{i !(k-i) !}(\rho(k-i))^{[l]} \\
& \left.+\left(\beta \alpha(t)+\alpha \frac{\psi}{1-\psi}\right)^{l+1} \sum_{i=0}^{l}(-1)^{i} \frac{\rho(l+1-i)+l}{i !(l+1-i) !}(\rho(l+1-i))^{[l]}\right\} .
\end{aligned}
$$

Thus

$$
\begin{aligned}
& { }_{\alpha} E\left(S_{N}-E S_{N}\right)^{r+1}=\theta^{-r-1} \sum_{l=1}^{r}\left(\begin{array}{l}
r \\
l
\end{array}\right)\left[-\rho\left(\beta t+\alpha \frac{\psi}{1-\psi}\right)\right]^{r-l} \\
& \quad\left\{\sum_{k=1}^{l}\left(\beta \alpha(t)+\alpha \frac{\psi}{1-\psi}\right)^{k} \sum_{i=0}^{k-1}(-1)^{i} \frac{\rho(k-i)+l-\rho\left(\beta t+\frac{\psi}{1-\psi}\right)}{i !(k-i) !}(\rho(k-i))^{[l]}\right. \\
& \left.+\left(\beta \alpha(t)+\alpha \frac{\psi}{1-\psi}\right)^{l+1} \sum_{i=0}^{l}(-1)^{i} \frac{\rho(l+1-i)+l}{i !(l+1-i) !}(\rho(l+1-i))^{[l]}\right\},
\end{aligned}
$$

which ends the proof.

Corollary 6.2. If $N \sim \operatorname{MNB}\left(\psi, t, \frac{\beta}{1+\beta}\right)$ and the i.i.d. summands $\left\{X_{i}\right.$, $i \geq 1\}$ have the gamma distribution (5.16), then

$$
\begin{aligned}
& { }_{\alpha} E S_{N}=\theta^{-1} \rho\left(\beta t+\frac{\psi}{1-\psi}\right) \\
& { }_{\alpha} E S_{N}^{2}=\theta^{-2}\left\{\beta t \rho^{[2]}+\beta^{2} t^{[2]} \rho^{2}+\frac{\psi}{1-\psi}\left[\rho^{[2]}+2 \beta t \rho^{2}+2 \frac{\psi}{1-\psi} \rho^{2}\right]\right\} \\
& { }_{\alpha} E S_{N}^{3}=\theta^{-3}\left\{\beta t \rho^{[3]}+3 \beta^{2} t^{[2]} \rho^{[2]} \rho+\beta^{3} t^{[3]} \rho^{3}\right. \\
& \left.+\frac{\psi}{1-\psi}\left[\rho^{[3]}+6 \beta t \rho^{[2]} \rho+3 \beta^{2} t^{[2]} \rho^{3}\right]+\left(\frac{\psi}{1-\psi}\right)^{2}\left[6 \rho \rho^{[2]}+6 \beta t \rho^{3}+6 \frac{\psi}{1-\psi} \rho^{3}\right]\right\}, \\
& { }_{\alpha} E S_{N}^{4}=\theta^{-4}\left\{\beta t \rho^{[4]}+\beta^{2} t^{[2]} \rho^{[2]} \rho(7 \rho+11)+6 \beta^{3} t^{[3]} \rho^{[2]} \rho^{2}+\beta^{4} t^{[4]} \rho^{4}\right. \\
& \quad+\frac{\psi}{1-\psi}\left[\rho^{[4]}+2 \beta t \rho^{[2]} \rho(7 \rho+11)+18 \beta^{2} t^{[2]} \rho^{[2]} \rho^{2}+4 \beta^{3} t^{[3]} \rho^{4}\right]
\end{aligned}
$$




$$
\begin{aligned}
& +\left(\frac{\psi}{1-\psi}\right)^{2}\left[2 \rho^{[2]} \rho(7 \rho+11)+36 \beta t \rho^{[2]} \rho^{2}+12 \beta^{2} t^{[2]} \rho^{4}\right] \\
& \left.+\left(\frac{\psi}{1-\psi}\right)^{3}\left[36 \rho^{[2]} \rho^{2}+24 \beta t \rho^{4}+24 \frac{\psi}{1-\psi} \rho^{4}\right]\right\} \\
& { }_{\alpha} \sigma^{2} S_{N}=\theta^{-2}\left\{\beta t \rho^{[2]}+\beta^{2} t \rho^{2}+\frac{\psi}{1-\psi}\left[\rho^{[2]}+\frac{\psi}{1-\psi} \rho^{2}\right]\right\}, \\
& { }_{\alpha} E\left(S_{N}-E S_{N}\right)^{3}=\theta^{-3}\left\{\beta t \rho^{[3]}+3 \beta^{2} t \rho^{[2]} \rho+2 \beta^{3} t \rho^{3}\right. \\
& \left.+\frac{\psi}{1-\psi}\left[\rho^{[3]}+3 \frac{\psi}{1-\psi} \rho^{[2]} \rho+2\left(\frac{\psi}{1-\psi}\right)^{2} \rho^{3}\right]\right\}, \\
& { }_{\alpha} E\left(S_{N}-E S_{N}\right)^{4}=\theta^{-4}\left(t \beta \rho^{[4]}+t \beta^{2}\left(3 t \rho^{[2]} \rho^{[2]}+7 \rho^{[2]} \rho^{2}+11 \rho^{[2]} \rho\right)\right. \\
& +6 \beta^{3}\left(t^{[2]}+t\right) \rho^{[2]} \rho+3 \beta^{4}\left(t^{[2]}+t\right) \rho^{4} \\
& +\frac{\psi}{1-\psi}\left[\rho^{[4]}+6 t \beta \rho^{[2]} \rho^{[2]}+6 t \beta^{2} \rho^{[2]} \rho^{2}\right] \\
& +\left(\frac{\psi}{1-\psi}\right)^{2}\left[10 \rho^{[2]} \rho^{2}+14 \rho^{[2]} \rho+6 t \beta \rho^{[2]} \rho^{2}+6 t \beta^{2} \rho^{4}\right] \\
& \left.+18\left(\frac{\psi}{1-\psi}\right)^{3} \rho^{[2]} \rho^{2}+9\left(\frac{\psi}{1-\psi}\right)^{4} \rho^{4}\right), \\
& V=(\beta t \rho+\psi \rho(1-\beta t))^{-1}\left(\beta t \rho^{[2]}+\beta^{2} t \rho^{2}+\psi\left(\rho^{[2]}-2 \beta t \rho^{[2]}-2 \beta^{2} t \rho^{2}\right)\right. \\
& \left.+\psi^{2}\left(\beta t \rho^{[2]}+\beta^{2} t \rho^{2}-\rho\right)\right)^{1 / 2}, \\
& \gamma={ }_{\alpha} E\left(S_{N}-E S_{N}\right)^{3} /\left({ }_{\alpha} \sigma^{2} S_{N}\right)^{3 / 2} \\
& \kappa={ }_{\alpha} E\left(S_{N}-E S_{N}\right)^{4} /\left({ }_{\alpha} \sigma^{2} S_{N}\right)^{2} \text {. }
\end{aligned}
$$

Assuming that $\rho=1$ in Theorem 6.1, we have the following theorem.

THEOREM 6.3. The moment generating function, the rth moments and the $(r+1)$ th central moments of the compound $\alpha$-modified negative binomial distribution with exponential summands are as follows

$$
\begin{aligned}
& { }_{\alpha} M_{S_{N}}(s)=\left[1+\beta\left(1-\left(1-\frac{s}{\theta}\right)^{-1}\right)\right]^{-t} \frac{1-\psi}{1-\psi(1-s / \theta)^{-1}}, \\
& { }_{\alpha} E S_{N}^{r}=\theta^{-r} \sum_{k=1}^{r}\left(\beta \alpha(t)+\alpha \frac{\psi}{1-\psi}\right)^{k} \sum_{i=0}^{k-1}(-1)^{i} \frac{1}{i !(k-i) !}(k-i)^{[r]}, \\
& { }_{\alpha} E\left(S_{N}-E S_{N}\right)^{r+1}=\theta^{-r-1} \sum_{l=1}^{r}\left(\begin{array}{l}
r \\
l
\end{array}\right)\left[-\left(\beta t+\frac{\psi}{1-\psi}\right)\right]^{r-l}
\end{aligned}
$$




$$
\begin{aligned}
& \cdot\left\{\sum_{k=1}^{l}\left(\beta \alpha(t)+\alpha \frac{\psi}{1-\psi}\right)^{k} \sum_{i=0}^{k-1}(-1)^{i} \frac{k-i-\frac{\psi}{1-\psi}-\beta t+l}{i !(k-i) !}(k-i)^{[l]}\right. \\
& \left.+\left(\beta \alpha(t)+\alpha \frac{\psi}{1-\psi}\right)^{l+1} \sum_{i=0}^{l}(-1)^{i} \frac{2 l+1-i}{i !(l+1-i) !}(l+1-i)^{[l]}\right\} .
\end{aligned}
$$

REMARK 6.4. For $\psi=0$ see [KPW, p. 306].

Corollary 6.5. If $N \sim \operatorname{MNB}\left(\psi, t, \frac{\beta}{1+\beta}\right)$ and the i.i.d. summands $\left\{X_{i}\right.$, $i \geq 1\}$ have the exponential distribution, then

$$
\begin{aligned}
& { }_{\alpha} E S_{N}=\theta^{-1}\left(\beta t+\frac{\psi}{1-\psi}\right) \\
& { }_{\alpha} E S_{N}^{2}=\theta^{-2}\left\{2 \beta t+\beta^{2} t^{[2]}+\frac{2 \psi}{1-\psi}\left[1+\beta t+\frac{\psi}{1-\psi}\right]\right\} \\
& { }_{\alpha} E S_{N}^{3}=\theta^{-3}\left\{6 \beta t+6 \beta^{2} t^{[2]}+\beta^{3} t^{[3]}+\frac{\psi}{1-\psi}\left[6+12 \beta t+3 \beta^{2} t^{[2]}\right]\right. \\
& \left.\quad+6\left(\frac{\psi}{1-\psi}\right)^{2}\left[2+\beta t+\frac{\psi}{1-\psi}\right]\right\} \\
& \quad+\frac{\psi}{1-\psi}\left[24+72 \beta t+36 \beta^{2} t^{[2]}+4 \beta^{3} t^{[3]}\right] \\
& \left.\quad+\left(\frac{\psi}{1-\psi}\right)^{2}\left[72+72 \beta t+12 \beta^{2} t^{[2]}\right]+\left(\frac{\psi}{1-\psi}\right)^{3}\left[72+24 \beta t+24 \frac{\psi}{1-\psi}\right]\right\} \\
& { }_{\alpha} \sigma^{2} S_{N}=\theta^{-2}\left\{2 \beta t+\beta^{2} t+\frac{\psi}{1-\psi}\left[2+\frac{\psi}{1-\psi}\right]\right\} \\
& { }_{\alpha} E\left(S_{N}-E S_{N}\right)^{3}=\theta^{-3}\left\{6 \beta t+6 \beta^{2} t+2 \beta^{3} t\right. \\
& +3 \beta^{4}\left(t^{[2]}+t\right)+\frac{\psi}{1-\psi}\left[24+24 t \beta+12 t \beta^{2}\right]+\left(\frac{\psi}{1-\psi}\right)^{2}\left[48+12 t \beta+6 t \beta^{2}\right] \\
& \left.\left.\left.+36\left(\frac{\psi}{1-\psi}\right)^{3}+9\left(\frac{\psi}{1-\psi}\right)^{4}\right), \quad+6+6 \frac{\psi}{1-\psi}+2\left(\frac{\psi}{1-\psi}\right)^{2}\right]\right\} \\
& { }_{\alpha} E\left(S_{N}-E S_{N}\right)^{4}=\theta^{-4}\left(24 t \beta+t \beta^{2}(12 t+36)+12 \beta^{3}\left(t^{[2]}+t\right)\right.
\end{aligned}
$$




$$
\begin{aligned}
& V=\frac{\left(2 \beta t+\beta^{2} t-2 \psi\left(2 \beta t+\beta^{2} t-1\right)+\psi^{2}\left(2 \beta t+\beta^{2} t-1\right)\right)^{1 / 2}}{\beta t+\psi(1-\beta t)}, \\
& \gamma={ }_{\alpha} E\left(S_{N}-E S_{N}\right)^{3} /\left({ }_{\alpha} \sigma^{2} S_{N}\right)^{3 / 2}, \quad \kappa={ }_{\alpha} E\left(S_{N}-E S_{N}\right)^{4} /\left({ }_{\alpha} \sigma^{2} S_{N}\right)^{2} .
\end{aligned}
$$

Putting $\rho=1$ and $t=1$ in Theorem 6.1 we have

THEOREM 6.6. The moment generating function, the rth moments and the $(r+1)$ th central moments of the compound $\alpha$-modified geometric distribution with exponential summands are given as follows:

$$
\begin{gathered}
{ }_{\alpha} M_{S_{N}}(s)=\left[1+\beta\left(1-\left(1-\frac{s}{\theta}\right)^{-1}\right)\right]^{-1} \frac{1-\psi}{1-\psi(1-s / \theta)^{-1}}, \\
{ }_{\alpha} E S_{N}^{r}=\theta^{-r} \sum_{k=1}^{r}\left(\alpha \beta+\alpha \frac{\psi}{1-\psi}\right)^{k} \sum_{i=0}^{k-1}(-1)^{i} \frac{1}{i !(k-i) !}(k-i)^{[r]}, \\
{ }_{\alpha} E\left(S_{N}-E S_{N}\right)^{r+1}=\theta^{-r-1} \sum_{l=1}^{r}\left(\begin{array}{l}
r \\
l
\end{array}\right)\left[-\left(\beta+\frac{\psi}{1-\psi}\right)\right]^{r-l} \\
\cdot\left\{\sum_{k=1}^{l}\left(\alpha \beta+\alpha \frac{\psi}{1-\psi}\right)^{k} \sum_{i=0}^{k-1}(-1)^{i} \frac{k-i-\frac{\psi}{1-\psi}-\beta+l}{i !(k-i) !}(k-i)^{[l]}\right. \\
\left.+\left(\alpha \beta+\alpha \frac{\psi}{1-\psi}\right)^{l+1} \sum_{i=0}^{l}(-1)^{i} \frac{2 l+1-i}{i !(l+1-i) !}(l+1-i)^{[l]}\right\} .
\end{gathered}
$$

REMARK 6.7. For $\psi=0$ see [KPW, p. 305].

Corollary 6.8. If $N \sim \operatorname{MG}\left(\psi, \frac{\beta}{1+\beta}\right)$ and the i.i.d. summands $\left\{X_{i}\right.$, $i \geq 1\}$ have the exponential distribution, then

$$
\begin{aligned}
{ }_{\alpha} E S_{N} & =\theta^{-1}\left[\beta+\frac{\psi}{1-\psi}\right], \\
\alpha E S_{N}^{2} & =2 \theta^{-2}\left[\beta^{[2]}+\frac{\psi}{1-\psi}\left(1+\beta+\frac{\psi}{1-\psi}\right)\right], \\
{ }_{\alpha} E S_{N}^{3} & =6 \theta^{-3}\left[\beta^{[2]}(1+\beta)+\frac{\psi}{1-\psi}(1+\beta)^{2}+\left(\frac{\psi}{1-\psi}\right)^{2}\left(2+\beta+\frac{\psi}{1-\psi}\right)\right], \\
{ }_{\alpha} E S_{N}^{4} & =24 \theta^{-4}\left[\beta^{[2]}(1+\beta)^{2}+\frac{\psi}{1-\psi}(1+\beta)^{3}+\left(\frac{\psi}{1-\psi}\right)^{2}\left(3+3 \beta+\beta^{2}\right)\right. \\
& \left.+\left(\frac{\psi}{1-\psi}\right)^{3}\left(3+\beta+\frac{\psi}{1-\psi}\right)\right],
\end{aligned}
$$




$$
\begin{aligned}
& { }_{\alpha} \sigma^{2} S_{N}=\theta^{-2}\left[\beta(2+\beta)+\frac{\psi}{1-\psi}\left(2+\frac{\psi}{1-\psi}\right)\right], \\
& { }_{\alpha} E\left(S_{N}-E S_{N}\right)^{3}=\theta^{-3}\left[\beta\left(6+6 \beta+2 \beta^{2}\right)\right. \\
& \left.+\frac{\psi}{1-\psi}\left(6+6 \frac{\psi}{1-\psi}+2\left(\frac{\psi}{1-\psi}\right)^{2}\right)\right] \\
& { }_{\alpha} E\left(S_{N}-E S_{N}\right)^{4}=\theta^{-4}\left[\beta\left(24+48 \beta+36 \beta^{2}+9 \beta^{3}\right)\right. \\
& +\frac{\psi}{1-\psi}\left(24+48 \frac{\psi}{1-\psi}+36\left(\frac{\psi}{1-\psi}\right)^{2}+9\left(\frac{\psi}{1-\psi}\right)^{3}\right) \\
& \left.+\frac{\psi}{1-\psi}\left(12 \beta^{2}+24 \beta+6 \beta^{2} \frac{\psi}{1-\psi}+12 \beta \frac{\psi}{1-\psi}\right)\right], \\
& V=\frac{\left(\beta(2+\beta)-2 \psi(\beta(2+\beta)-1)+\psi^{2}(\beta(2+\beta)-1)\right)^{1 / 2}}{\beta+\psi(1-\beta)}, \\
& \gamma={ }_{\alpha} E\left(S_{N}-E S_{N}\right)^{3} /\left({ }_{\alpha} \sigma^{2} S_{N}\right)^{3 / 2}, \quad \kappa={ }_{\alpha} E\left(S_{N}-E S_{N}\right)^{4} /\left({ }_{\alpha} \sigma^{2} S_{N}\right)^{2} \text {. }
\end{aligned}
$$

Corollary 6.9. If $N \sim G\left(\frac{\beta}{1+\beta}\right)$ and the i.i.d. summands $\left\{X_{i}, i \geq 1\right\}$ have the exponential distribution, then

$$
\begin{aligned}
& E S_{N}=\theta^{-1} \beta, \quad E S_{N}^{2}=2 \theta^{-2} \beta^{[2]}, \quad E S_{N}^{3}=6 \theta^{-3} \beta^{[2]}(1+\beta), \\
& E S_{N}^{4}=24 \theta^{-4} \beta^{[2]}(1+\beta)^{2}, \\
& E\left(S_{N}-E S_{N}\right)^{2}=\theta^{-2} \beta(2+\beta), \\
& E\left(S_{N}-E S_{N}\right)^{3}=\theta^{-3} \beta\left(6+6 \beta+2 \beta^{2}\right), \\
& E\left(S_{N}-E S_{N}\right)^{4}=\theta^{-4} \beta\left(24+48 \beta+36 \beta^{2}+9 \beta^{3}\right), \\
& V=\frac{(\beta(2+\beta))^{1 / 2}}{\beta}, \quad \gamma=\frac{6+6 \beta+2 \beta^{2}}{\beta^{1 / 2}(2+\beta)^{3 / 2}}, \quad \kappa=\frac{24+48 \beta+36 \beta^{2}+9 \beta^{3}}{\beta\left(4+4 \beta+\beta^{2}\right)} .
\end{aligned}
$$

Acknowledgements. We are most grateful to Prof. J. L. Teugels for sending us copies of the Delaporte papers and for his lectures on insurance topics given in Lublin. Moreover, we thank the reviewer for suggestions improving the first version of this paper.

\section{References}

[BJ] S. Berg and J. Jaworski, Modified binomial and Poisson distributions with applications in random mapping theory, J. Statist. Plann. Inference 18 (1988), 313-322.

[BN] S. Berg and K. Nowicki, Statistical inference for a class of modified power series distributions with applications to random mapping theory, J. Statist. Plann. Inference 28 (1991), 247-261. 
[C] S. Chakraborty, On some new $\alpha$-modified binomial and Poisson distributions and their applications, Comm. Statist. Theory Methods 37 (2008), 1755-1769.

[CF] P. C. Consul and F. Famoye, Lagrangian Probability Distributions, Birkhäuser, Boston, 2006.

[D1] P. J. Delaporte, Quelques problèmes de statistiques mathématiques posés par l'assurance automobile et le bonus pour non sinistre, Bull. Trimest. Inst. Actuar. Français 227 (1959), 87-102.

[D2] P. J. Delaporte, Un problème de tarification de l'assurance accidents d'automobiles examiné par la statistique mathématique, in: Trans. XVIth Int. Congr. Actuaries (Brussels, 1960), Vol. 2, 121-135.

[DM] P. J. Diggle and R. K. Milne, Negative binomial quadrat counts and point processes, Scand. J. Statist. 10 (1983), 257-267.

[FKS] H. Finner, P. Kern and M. Scheer, On some compound distributions with Borel summands, Insurance Math. Econom. 62 (2015), 234-244.

[G] A. Gut, Probability: A Graduate Course, Springer, New York, 2005.

[GT] R. W. Grubbström and O. Tang, The moments and central moments of a compound distribution, Eur. J. Operational Research 170 (2006), 106-119.

[J] J. Jaworski, Random mappings and Abel sums, in: Contributions to Social Network Analysis, Information Theory and Other Topics in Statistics-Festschrift in honor of Ove Frank (J. Hagberg, ed.), Stockholm, 2002, 226-243.

[JKK] N. Johnson, A. Kemp and S. Kotz, Univariate Discrete Distributions, 3rd ed., Wiley, Hoboken, NJ, 2005.

[KPW] S. A. Klugman, H. H. Panjer and G. E. Willmot, Loss Models: From Data to Decisions, Wiley, New York, 1998.

[P] H. H. Panjer, Discrete parametric distributions, in: Encyclopedia of Actuarial Science, J. L. Teugels and B. Sundt (eds.), Vol. 1, Wiley, New York, 2006.

[PW] H. H. Panjer and G. E. Willmot, Insurance Risk Models, Soc. Actuaries, Schaumburg, IL, 1992.

[R] J. Riordan, Combinatorial Identities, Wiley, New York, 1968.

[SS1] K. Steliga and D. Szynal, On formulae for central moments of counting distributions, Appl. Math. (Warsaw) 42 (2015), 111-124.

[SS2] K. Steliga and D. Szynal, On the compound $\alpha(t)$-modified Poisson distribution, Appl. Math. (Warsaw) 42 (2015), 359-377.

[SV] B. Sundt and R. Vernic, Recursion for Convolutions and Compound Distributions with Insurance Applications, Springer, Berlin, 2009.

[V] D. Vose, Risk Analysis. A Quantitative Guide, Wiley, 2008.

[W] K. H. Wehrhahn, Combinatorics. An Introduction, 2nd ed., Carslaw Publ., Glebe, 1992.

[WN] C. S. Withers and S. Nadarajah, On the compound Poisson-Gamma distribution, Kybernetika 47 (2011), 15-37.

Katarzyna Steliga

Department of Mathematics

Lublin University of Technology

Nadbystrzycka 38A

20-618 Lublin, Poland

E-mail: k.steliga@pollub.pl
Dominik Szynal Institute of Mathematics Maria Curie-Skłodowska University pl. M. Curie-Skłodowskiej 1 20-031 Lublin, Poland E-mail: szynal@poczta.umcs.lublin.pl 